\title{
Overview on 5G Radio Frequency Energy Harvesting
}

\author{
Sanae El Hassanit ${ }^{7}$, Hind El Hassani ${ }^{2}$, Noureddine Boutammachte ${ }^{3}$ \\ ${ }^{1}$ LTI Laboratory, ENSA El Jadida, Chouaib Doukkali University, 24 000, Morocco \\ ${ }^{2}$ LISA Laboratory, ENSA Fès, Sidi Mohamed Ben Abdellah University, 30 000, Morocco \\ 3 3ER Team, ENSAM Meknès, Moulay Ismail University, 50 000, Morocco
}

A R T I C L E I N F O

Article history:

Received: 21 May, 2019

Accepted: 02 August, 2019

Online: 19 August, 2019

Keywords:
Energy Harvesting
$5 G$
Green technologies
SWIPT
Rectenna

\section{Introduction}

This paper is an extension of the overview originally presented in 2017 about $R F$ Energy Harvesting For $5 G$ at the International Renewable and Sustainable Energy Conference (IRSEC) [1]. In fact, due to the rapid evolution of new technologies, the world faces new challenges concerning Energy Efficiency (EE). The transformation in users habits, driven by the installation of new services and the creation of new needs, especially in telecommunication, resulted in a significant rising in volume demand and requirements diversity. These transformations are leading the development of the fifth Generation (5G) of mobile systems, expected by 2020 [2], and research is being conducted to issue this new generation. In addition to the growing number of subscribers, new applications as for smart cities, smart grids, Internet of Things (IoT) and Machine-2-Machine (M2M) communications imply an increasing number of sensors, and thus the need of reviewing the system design. The sophisticated power hungry future handsets, the very large number of simpler sensors, and the dense network architecture necessary to respond to the growing needs are all factors to make energy management an urgent issue for future systems. EE appears thus as a major development key of $5 \mathrm{G}$ systems, as pointed in many $5 \mathrm{G}$ visions by telecommunication industry actors [3, 4, 5]. In order to enhance the energy consumption and management, techniques like Radio-Frequency Energy Harvesting (RF-EH), virtualized network functionalities, cloud technologies, etc. are proposed.

Energy aspects in 5G systems have attracted a lot of attention in the last years, and many studies, reviews and tutorials have considered these aspects. For example, in [6], authors provide a survey on energy-efficient wireless communications, with a discussion on the most relevant challenges at that time. General energy efficient technologies are discussed iwith no specific interest in EH solutions. Authors in [7] give an overview of EH techniques, in particular on EH power management policies optimizations, with specific stress on Wireless Sensor Networks (WSNs) and on ambient RF harvesting. In their survey [8], authors discuss $5 \mathrm{G}$ green network EE and promising technologies and architecture for its optimizations. Among these techniques, EH is discussed as well as other key enabling techniques, but specific EH integration is not detailed. The authors in [9] also consider EH from a circuit design point of view, particularly for embedded systems, and authors in [10] research RF-EH for IoT from an implementation perspective, including the design of antennas, rectifiers, and matching networks.

This paper aims to give a general review of EH in $5 \mathrm{G}$ systems, which includes both ambient and dedicated EH. In addition, considering the imminent commercial launching of 5G, and the ongoing standardization of 5G New Radio (5GNR) [11], an updated overview of RF-EH techniques is needed taking into account the standardization landscape. To this end, $5 \mathrm{G}$ requirements and use cases, as well as $5 \mathrm{G}$ ecosystem and some of the key $5 \mathrm{G}$ enabling technologies, are

*Corresponding Author: Route d'Azzemour Nationale N1 ELHAOUZIA BP 1166 El Jadida 24002 Morocco, (+212) 523344822 \& elhassani.s@ucd.ac.ma 
presented in the first section. In addition, a special subsection is dedicated to IoT, as one of the most RF-EH appealing $5 \mathrm{G}$ enabled use cases. The second section discusses EE in $5 \mathrm{G}$ networks from both network and terminal sides, while RF-EH implementation challenges and candidate solutions are reviewed in the the third section. The fourth section presents RF-EH in 5G ecosystem, giving a literature review of combinations between RF-EH and some of the most important $5 \mathrm{G}$ key enabling technologies. A conclusion of the paper is then given.

\section{$25 G$ general technological landscape}

\subsection{G Requirements}

Compared to current mobile generation, 5G mobile data rate volume per area is expected to be multiplied by 1000 , and user data rate as well as the number of connected devices are expected to be multiplied by 10 to 100 . Battery life should also be 10 times longer, and latency 5 times shorter. 5G requirements are summarized in Figure 1 and the International Telecommunication Union (ITU), which has established an R\&D programme worldwide on International Mobile Telecommunications for 2020 and beyond values(IMT-2020), has established a 5G framework as illustrated in Table 1][12].

Table 1: $5 \mathrm{G}$ requirements

\begin{tabular}{|c|c|}
\hline Requirements & Desired Value \\
\hline \hline Data Rate & 1 to 10 Gbps \\
\hline Data Volume & $\begin{array}{c}\text { 9 GB/h (busy period) } \\
\text { and 500GB/month/user }\end{array}$ \\
\hline Latency & Less than 5 ms \\
\hline Battery Life & One decade \\
\hline Connected devices & 300000 devices/AP \\
\hline Reliability & $99.999 \%$ \\
\hline
\end{tabular}

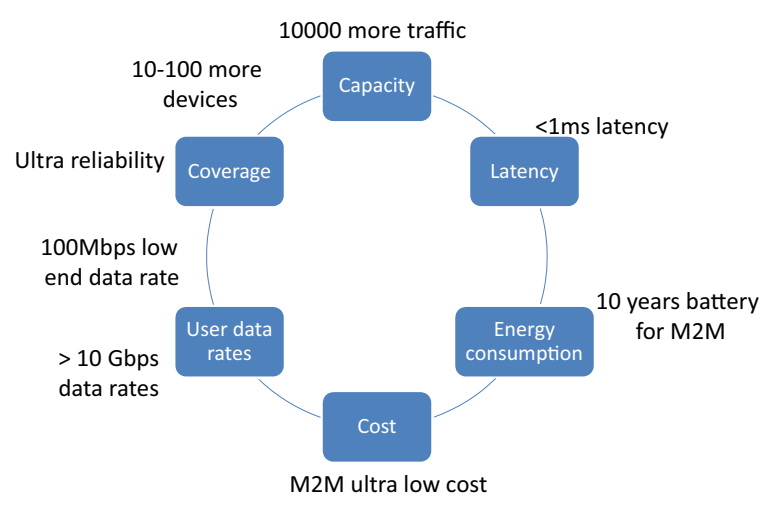

Figure 1: 5G key requirements summary (Source [13])

5G systems are meant to manage at the same time very low cost devices, such as sensors, and advanced ones, such as smartphones and tablets. For example, simple devices may choose not to use large bands. Thus, in the same system, and in some frequency bands, smart devices will use a band of $100 \mathrm{MHz}$ while sensors only $10 \mathrm{MHz}$. Note that $5 \mathrm{G}$ will have to manage much denser networks than today, around 1 million connections $/ \mathrm{km}^{2}$, and the more predominant design parameter will thus be the cost/area.

\section{2 $5 \mathrm{G}$ use cases}

Traditional as well as new use cases are addressed in 5G, and this implies new challenges. The corresponding requirements are specified by several organizations and research actions.

Each use case has its own requirements, such as throughput, delay, reliability, etc. and consequently uses different techniques. Hence, a wide range of devices, such as smartphone, wearable, MTC, etc., are being all expected to use $5 \mathrm{G}$ systems in a diverse heterogeneous environment. A categorization example of use cases has been developed by the Next Generation Mobile Networks (NGMN) Alliance through representative examples, which leads to 8 families of use cases [14], illustrated in Figure 2]
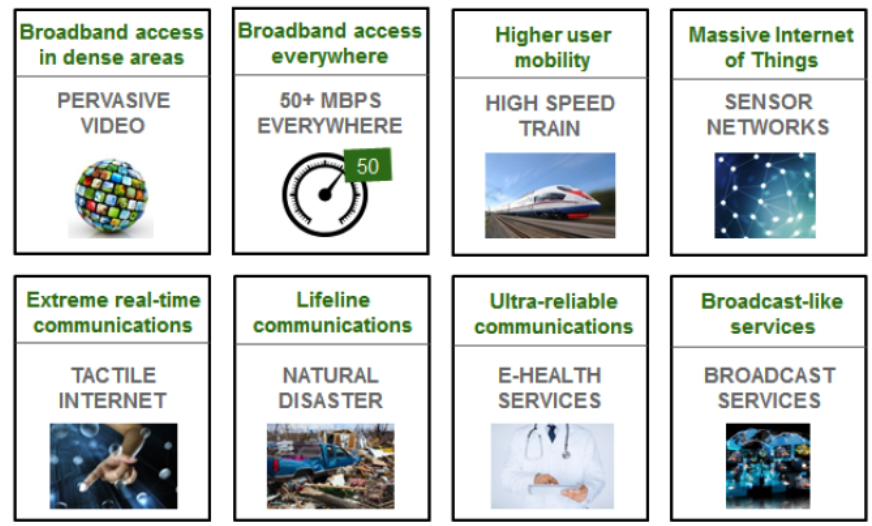

Figure 2: Families of use cases identified by NGMN (Source[14])

For example, smart cities applications including metering, city or building lights management, environment monitoring, and vehicle traffic control can be deployed as WSNs. These services, when aggregated, form a high density devices environment with heterogeneous technologies that need to be managed in a common communication and interworking framework, where very low cost devices with very long battery life may exist. 5G may provide platform for such deployments. Another example of $5 \mathrm{G}$ use cases is the mobile Video Surveillance, which is expected to be deployed almost everywhere. This means an automated analysis of the video footage with constraints, though relatively reduced for battery life, but high for reliability and security of the network as well as performance and interactivity with remote systems.

Note that the 3rd Generation Partnership Project (3GPP) issued and approved four Technical Reports on 2016, which outlined new Services and Markets Technology Enablers (SMARTER) [15], and contained more than 70 different use cases categorized into different groups :

- Massive Internet of Things, particularly relevant to the new vertical services, such as smart home and city, smart utilities, e-Health, and smart wearables.

- Critical Communications, for which there is more need for latency, reliability, and availability. Use case 
examples are industrial control applications and tactile Internet.

- Enhanced Mobile Broadband, including use case families related to higher data rates, higher density, deployment and coverage, higher user mobility, devices with highly variable user data rates, fixed mobile convergence, and small-cell deployment.

- Network Operation, addressing functional system requirements, including for example flexible functions and capabilities, new value creation, migration and interworking, optimizations and enhancements, and security.

Example of projects exploring EE and EH in 5G systems is Sustainable Cellular Networks Harvesting Ambient Energy (SCAVENGE) [16], which is a project funded from the European Union's Horizon 2020. It defines among others an architecture framework for EH networks for Core Network $(\mathrm{CN})$ and Radio Access Network (RAN).

\subsection{G ecosystem}

$5 \mathrm{G}$ systems cover a large span of use cases, with different data rates, latencies, reliabilities, densities, required powers, etc. This implies many tradeoffs, and a highly scalable architecture. As described in [17], the new 5G ecosystem is complex and involves many participants, including manufacturers for components, chipsets, network equipments and wireless devices, in addition to mobile networks operators, application developers and even users themselves, all overlaid by different standardization entities. As stated in [18], the first mover of $5 \mathrm{G}$ is the wireless part as it establishes foundations for the feasibility of final products and services. Many connectivity options are considered in 3GPP, which allows several deployment alternatives. However, as highlighted in [18] the focus for initial deployments is on non-standalone NR and standalone NR, as the deployment of several options may make the whole $5 \mathrm{G}$ ecosystem too complex. The simplification of $5 \mathrm{G}$ through reduction of deployment options is also adopted by other industry actors, such as Ericsson [19].

\subsection{Examples of 5G key enabling technolo- gies}

$5 \mathrm{G}$ systems require an important change in the cellular architecture as well as key wireless technologies. The requirement for massive network capacity along with the increased user experience suggest research in numerous dimensions. In particular, the RAN appears as one of the biggest challenges. For example, the increase in bandwidth demand implies the optimization of current systems spectrum use, unlicensed spectrum use and the allocation of new bands, starting with the move to millimeter wave (mmWave) bands. The optimization of spectrum use also means that, among others, new waveforms and advanced Multiple Input Multiple-Output (MIMO) will be used. Extreme densification and offloading are also key elements to enhance capacity, which implies an urgent need for virtualization and softwarization of the network. As presented in [4], the Figure 3 illustrates these needs in three distinct dimensions.

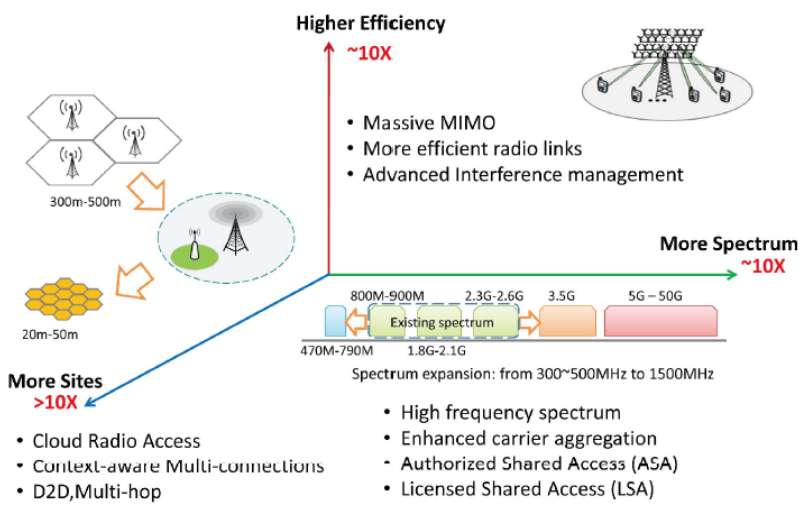

Figure 3: 5G technological research in three different dimensions (Source: [4])

Examples of key $5 \mathrm{G}$ enabling technologies are given in the remainder of this subsection.

\subsubsection{Spectrum allocation}

$5 \mathrm{G}$ is a convergence of previous and new systems, and its spectrum is expected to be a combination of allocated and new bands. Some of the spectrum below $6 \mathrm{GHz}$ is thus being re-purposed for use with newer technologies; it is the primary $5 \mathrm{G}$ band, as decided in WRC 2015. Complementary $5 \mathrm{G}$ bands above $6 \mathrm{GHz}$, are expected to be allocated in the 2019 World Radiocommunication Conference (WRC 2019) [20] (October). Illustration of these bands is given in Figure 4. On the The 3rd Generation Partnership Project (3GPP) Release-15, the New Radio-Unlicensed spectrum (NR-U) work item supports both the existing $5 \mathrm{GHz}$ unlicensed band and the new "greenfield" $6 \mathrm{GHz}$ unlicensed band. Other unlicensed and shared spectrum bands, including mmWave, can be expected in coming releases [11], even though it is not as mature as current frequency bands. In fact, mmWave has rather hostile propagation characteristics, as well as a current comparatively high equipment cost, which has limited its use so far. However, 5G mmWave mobile technology have been already demonstrated, as first by Samsung in May 2013 [21], and later by others, as for Huawei [22]. A more accomplished $5 \mathrm{G}$ pilot system was demonstrated in 2018 Winter Olympics [23].

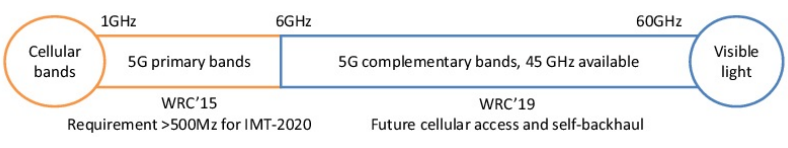

Figure 4: WRC-15 and WRC-19 5G spectrum allocation

Integration of many bands for the same transmission can be made through Cognitive Radio (CR), motivated by the underutilization of many frequency bands. In fact, CR allows dynamic allocation of underutilized resources, after their sensing, in an opportunistic way without altering the other users, and thus to expand the spectrum of a cellular networks on demand and at a relatively low cost [24, 25]. 


\subsubsection{Carrier Aggregation (CA)}

As $5 \mathrm{G}$ is a convergence of many systems, the $5 \mathrm{G}$ radio access uses simultaneously many technologies, with possibly different frequency bands. This may enable a more effective use of the crowded and fragmented spectrum, a simultaneous use of different Radio Access Technologies (RATs) and more flexibility of resource allocation. However, this implies also the need to aggregate different carriers. CA is already used in Long Term Evolution (LTE) since 3GPP Release 10 for inter and intra LTE-LTE contiguous carriers aggregation. Release 11 presented Intra-band non-contiguous CA, and Release 12 presented CA for Frequency Division Duplex (FDD) and Time Division Duplex (TDD). Release 13 presented CA for $5 \mathrm{GHz}$ WiFi bands, called Licensed Assisted Access, and Release 14, which starts 5G standardization, defines more cases following the $5 \mathrm{G}$ adopted bands [26]. Some issues should be considered for Internet Protocol (IP) configuration, interference coordination and time synchronization. Moreover, resource scheduling over the aggregated bands should be optimized and may use advanced tools, such as game theory [27].

\subsubsection{Massive MIMO}

Many techniques used to enhance spectrum efficiency are surviving in $5 \mathrm{G}$, while other new techniques are proposed. MIMO antennas techniques is already used for LTE and LTE-Advanced (LTE-A), and 5G proposes massive MIMO as one of its most important enabling techniques. In fact, thanks to multiple antennas at the Base Station (BS), the energy in downlink can be focused to form beams towards the User Equipment (UE). In 5G, a large number of antennas, i.e. massive MIMO, can enable the serving of many users in an accurate way. Moreover, when combined with other $5 \mathrm{G}$ techniques, like small cells and mmWave, it provides significant capacity, as required for $5 \mathrm{G}$ systems [28]. Note that massive MIMO can also be used for backhauling [29].

\subsubsection{Channel modeling}

In $5 \mathrm{G}$ systems, the expected bands to be used, especially above $6 \mathrm{GHz}$, are not addressed by current models, and accurate models are needed to enable many of the physical layer techniques. Thus, research is already conducted for mmWave characterization in several environments [30]. As indicated in [31], the new channel model extends the existing 3GPP 3D channel model, and handles frequencies up to 100 $\mathrm{GHz}$ in several mobility scenarios. In this optic, 3GPP RAN Meeting for example was held in Busan Korea in June 2016 and approved the first standard for the mobile broadband $5 \mathrm{G}$ high-frequency $(6-100 \mathrm{GHz})$ channel model.

\subsubsection{Signal waveforms}

Multicarrier modulation, particularly Orthogonal Frequency Division Multiplexing (OFDM), is adopted in LTE and enables good spectrum efficiency. However, it still presents some drawbacks, as it inserts a Cyclic Prefix (CP) that reduces the efficiency, in addition to its large sidelobes, and thus considerable Out-Of-Band (OOB) emission, resulting from the rectangular pulse shaping,as well as its high Peak to Average Power Ratio (PAPR). Most popular candidates waveform for $5 \mathrm{G}$, in addition to OFDM, include :

- Filtered OFDM, which removes OOB emissions by filtering the signal [32].

- Filter Bank based MultiCarrier-Offest Quadrature Amplitude Modulation (FBMC-OQAM), which is a modified OFDM requiring no $\mathrm{CP}$ and thus enhancing spectrum efficiency [33]. Sub-carriers are filtered at both transmitter and receiver sides, and this reduces OOB and makes the signal more robust against Doppler effect, Inter-Symbol Interference (ISI), synchronization imperfections, spectrum fragmentation, etc. This also results in self-equalization, which can reduce the number of unused subcarriers, and blind channel tracking, reducing pilot contamination and thus enhancing massive MIMO functioning.

- Universal Filtered Multi Carrier (UFMC), which filters subbands rather than subcarriers [34]. This gives more flexibility to the design. The use of a prefix in this case depends on filters design.

- Generalized Frequency Division Multiplexing (GFDM), which also provides a flexible structure via adjustable pulse shaping and subcarrier filtering. In addition, data symbols are grouped both in time and frequency domains. However, filtering flexibility implies self-created interference, which is dealt with using iterative interference cancellation at the receiver [35].

\subsubsection{Multiple access}

The multiple access technique used in 4G is OFDMA, which may survive for $5 \mathrm{G}$. However, massive MIMO in a dense network context, multi-user MIMO (MU-MIMO) can be enabled using Spatial Division Multiple Access (SDMA). For higher capacity, Non-Orthogonal Multiple Access (NOMA) schemes are also proposed for $5 \mathrm{G}$, as it allows more than one user to share the same subband without coding or spreading redundancy [36, 37, 38]. For this scheme, users are superposed in time and frequency, and have different powers following their channel conditions. Differentiation of the users is then performed using Successive Interference Cancellation (SIC), which uses joint processing combined with subcarrier and power allocation algorithm [39, 40]. An illustration of NOMA mechanism is given in Figure. 5. So far, 3GPP NR has specified flexible technology framework that can be tuned to enable a wide range of $5 \mathrm{G}$ scenarios, and more specifications are expected for Release 16 [41].

\subsubsection{Ultra Dense Network (UDN)}

$5 \mathrm{G}$ brings a substantial network architecture redesign, particularly through densification, in order to support the expected heavy traffic. Networks densification has been used since the second generation $(2 \mathrm{G})$ for capacity scaling, but the ultra densification for future networks can provide an extreme and user-centric reuse of system bandwidth on the spatial domain, and improve propagation conditions by reducing the distance between the end user and the BS. It implies 
an increased spatial reuse of system resources, a large node density and irregular deployments. Challenging issues about UDN include among others interference management, heterogeneous multi-RAT optimization, the possibly high cost of a dense infrastructure, the cell-edge effect, and the backhauling.

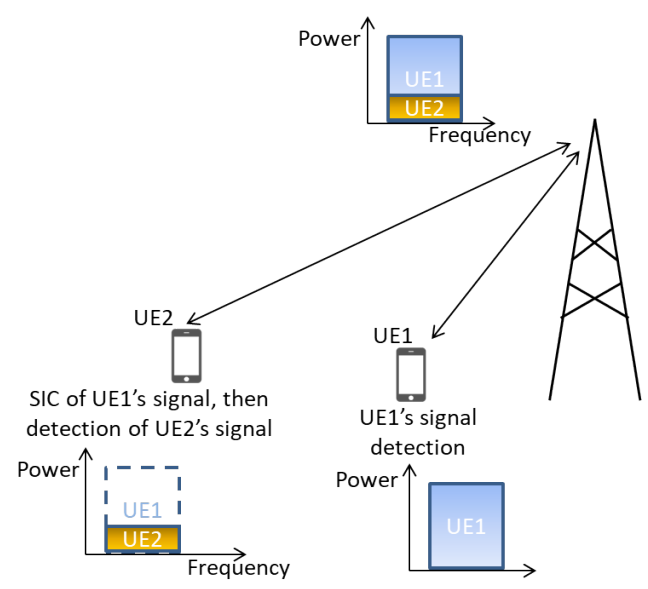

Figure 5: Downlink NOMA in a simple scenario with one BS and two UEs

\subsubsection{Cloud-Based Radio Access Network (C-RAN)}

As network densification is a key $5 \mathrm{G}$ networks enabler, it is expected that BSs will have to handle a heavy radio processing. A suggested solution in this case is the Cloudbased RAN (C-RAN). In fact C-RAN dissociates the BaseBand processing Unit (BBU) from the Remote Radio Heads (RRHs); For resource optimization, the BBU part is moved to a pool, or to the cloud, which is a virtualized cluster connected to the core network through the backhaul, and the transmission of the radio signal is performed by RRHs based on computed signals from the cloud. On the otehr hand, RRH is connected with the cloud BBU pool via a fronthaul.

RRHs are cheaper and simpler if cloud based computations are used. This allows thus more scalability and densification opportunities. It can also reduce the delay for inter-cell coordination and permits more joint optimization of processing, e.g. for cooperative interference management, scheduling, Coordinated Multi-Point, etc. [42].

C-RANs can also be self-organizing; intelligent smart cells are deployed in the area covered by a macro BS, and can enable advanced clustering and coordination schemes to enhance mobility and handovers. In addition, C-RAN in Heterogenous context lead to H-CRAN, where different types of nodes, including low power and high power nodes, use the same computing pools. The H-CRAN is illustrated in Fig. 6 .

\subsubsection{Network architecture}

5G cellular networks are expected to be multi-RAT and multi-layer. Considering emerging $5 \mathrm{G}$ use cases, current network architecture is relatively incompetent to address $5 \mathrm{G}$ requirements. Thus, one of the central objectives in $5 \mathrm{G}$ architecture is network flexibility. Logical core and RAN have to be split to allow their evolution and to ease their deployment. To this end, the European Telecommunications Standards
Institute (ETSI) adopted the separation of User Plane (UP) functions and Control Plane (CP) functions, and modularization of the function design, e.g. to enable flexible and efficient network slicing [44]. Similarly, a 5G architecture made of three layers and a management entity is presented by NGMN [45]. It is based on a central cloud and typically comprises multiple data centres which may be very distant, and connected to each other or to the central cloud or edge clouds. Figure 7 illustrates this topology as viewed by the project 5G NOvel Radio Multiservice Adaptative network architecture (5G NORMA), which is one of the 5G Public Private Partnership (5G PPP) projects under the Horizon 2020 framework [45].

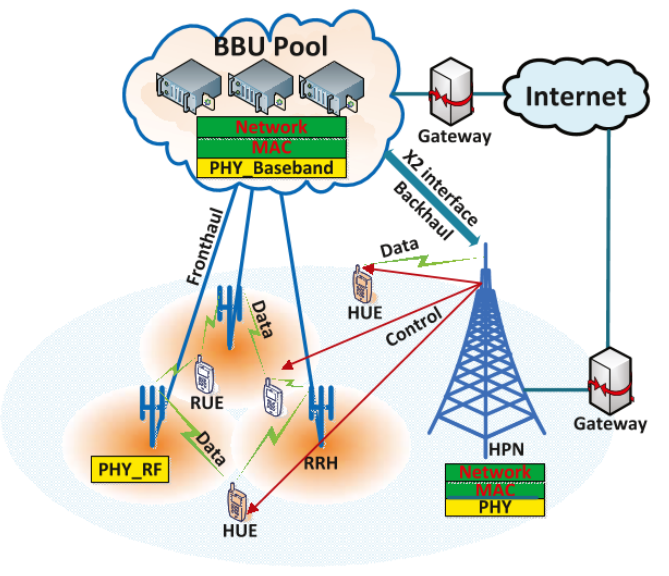

Figure 6: H-CRAN : Heterogeneous Cloud Radio Access Networks (Source [43])

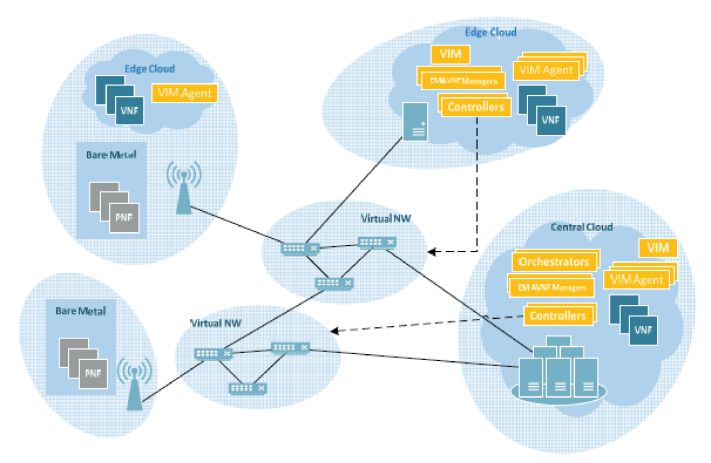

Figure 7: 5G NORMA topology view on architecture

As suggested in [46], in order to avoid redundancy, low layer functionalities are insured by radio logical network while a network cloud provides all higher layer functionalities. In addition, functions can be dynamically deployed in the network cloud through Software Defined Networks/Network Functions Virtualization (SDN/NFV).

In addition to layers, $5 \mathrm{G}$ uses network slicing to deliver services with different performance characteristics. It is a network technique, initially for the core network but extended to an End-to-End (E2E) concept including also RAN part. For example, massive IoT service, connecting fixed sensors that measure humidity, temperature, etc. to mobile networks, does not require advanced mobility features, critical in serving mobile phones. In contrary, a mission-critical IoT service, like autonomous driving or remote controlled 
robots, requires a very low E2E latency. To avoid building a dedicated network for each service, multiple logical networks over a single physical network can be insured through network slicing. The physical network is sliced up into different virtual E2E slices, with specific network functions and RAT parameters, following the requirements of the use case or business model. Note that network slicing also limits error propagation between slices.

\subsubsection{NFV/SDN}

An important trend for $5 \mathrm{G}$ networks is virtualization, as it enables resource sharing. In addition, hardware and software have to be dissociated, and network functions to be moved towards software. Assuming separation between control and data, NFV is an important $5 \mathrm{G}$ architecture driver. It provides network adaptability and scalability [44], boosted by automated mechanisms; simple and fast operations will be needed to deploy new network features, and resources can be easily shared through the abstraction of physical resources to a number of virtual resources. An illustration of wireless network virtualization is given Figure 8

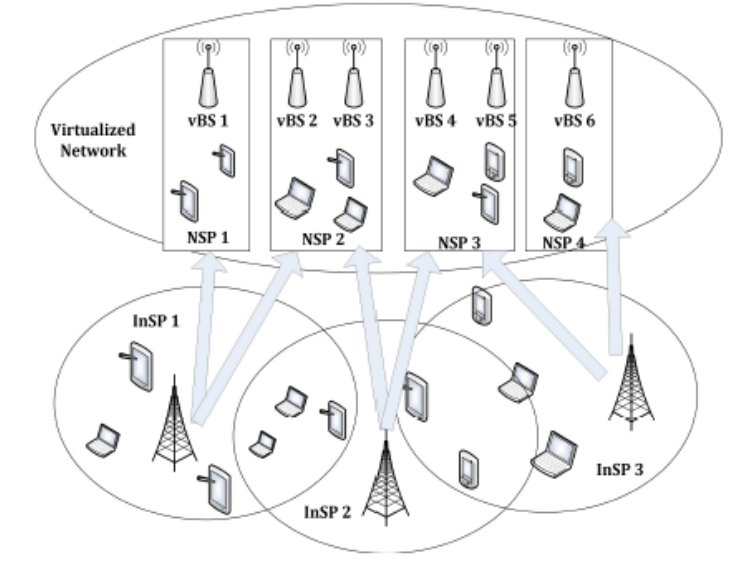

Figure 8: An example of wireless network virtualization (Source 47])

NFV uses a virtualized commercial server to set up network function software in virtual machines. RAN works thus as edge cloud while core works as core cloud. Connectivity among virtual machines located in edge and core clouds is insured by SDN. This makes thus the network architecture ultra-flexible; NFV processes network functions in the cloud, and SDN creates and exposes private virtual networks, linking thus the cloud back to the network. SDN enables the orchestration of the NFV physical and virtual infrastructure resources, through supporting provisioning and configuration of network connectivity and bandwidth.

\subsubsection{Mobile Edge Computing}

The diversity of $5 \mathrm{G}$ applications and the density of the network may lead to congestion, which may be solved by Mobile Edge Computing (MEC), mainly standardized by ETSI, 3GPP and ITU-T. MEC provides Information Technologies (IT) and cloud computing functions in the RAN, in close proximity to mobile subscribers. This enhances responsiveness to content, services and application requests, and en- ables thus ultra-low latency, high bandwidth radio network information.

MEC introduces new processing capabilities in the BS for new applications, with a new functions separation and a new interface between BBU and Remote Radio Unit (RRU). This is linked to C-RAN as C-RAN implements baseband processing capabilities in distributed locations. A demonstration for a $5 \mathrm{G}$ MEC proof-of-concept was conducted by Ericsson and Vodafone [48].

Note that MEC is currently deployed in LTE networks as an add-on to offer services in the edge. However, 5G system specifications provide new functionalities for edge computing [44].

\subsubsection{Device to Device communications (D2D)}

High-density expected for $5 \mathrm{G}$ networks can also be solved by D2D communication. In fact, unlike voice-centric systems, where no spatial proximity is assumed between communication parties, some data communication cases may imply collocated devices which try to wirelessly share content. In this case, it can be more efficient to use proximity-based D2D communication, which may also enhance capacity and coverage when devices act as transmission relays. Note that D2D is already used in $4 \mathrm{G}$, and presents some challenges for 5G. Figure 9 presents typical downlink D2D scenario.

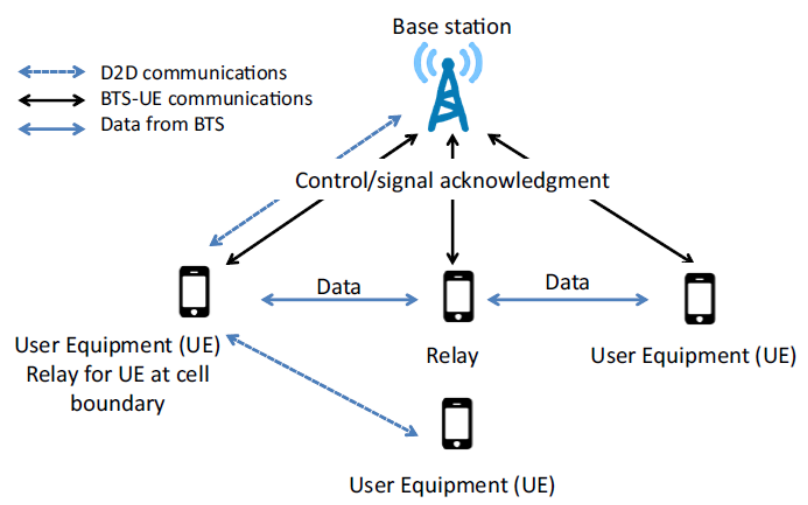

Figure 9: D2D typical scenario (Source [49])

\subsection{IoT applications landscape}

\subsubsection{IoT characteristics}

In IoT applications, a large variety of devices is implied, from small devices to big data centres. The devices can be self-empowered, battery-empowered or connected to the grid. For IoT specific applications, most devices, in number, are battery-empowered or self empowered, i.e. using EH.

IoT is distinct from WSN as it has specific characteristics. In particular, IoT energy sources have the following characteristics [50]:

- Scalability, as IoT often imply large deployments in possibly variable locations with different accessibilities. In this case, batteries are not ideal.

- Maintenance-free, as the deployments often imply a large number of devices. Wired empowering would be thus impossible and battery-based one would be costly. 
- Mobility support, as for many applications, the devices are mobile.

- Long life-time, as in some applications, and because of difficult maintenance, devices are supposed to work autonomously up to several decades.

- Flexibility, as capacity and size of energy sources may vary following the IoT application.

- Low-cost, as IoT are usually deployed in a large scale, which means that a small difference in the unit cost may limit affordability of the application.

- Sustainability, as large scale deployments may imply significant power consumption, requiring thus sustainable power solutions.

- Environment-friendly, as IoT popularity may imply serious environmental impact, especialy if disposable batteries are used for devices powering.

Currently, solutions meeting these characteristics with very low power consumption are standardized, but having energy autonomy is even more attractive. EH-enabled nodes techniques, as well as batteries storing harvested energy and optimized low power circuits, have to be thus combined with power saving mechanisms and network resource management. Note that for traditional wireless networks, many factors are important in the design. For example, the acceptable latency of the transmission can lay from some milliseconds to some days. Transmission rate is also generally low, mostly some kilobytes per second. Similarly, the distance between nodes is critical, as it contributes in the definition of the transmission power. Depending on the application, the distance can range from centimeters to kilometers, and it can vary over time when mobility of the nodes is considered.

\subsubsection{IoT standards and solutions}

RF-EH globally provides little energy compared to other energy sources. Thus, it is more suitable for low power IoT applications. These are hence one of the most interesting motivations for RF-EH technologies development. It is also one of the vectors in international standardization bodies. Even though solutions exist since many years for low-power applications, including Wi-Fi, Bluetooth, and ZigBee, these are unsuitable for long-range cellular and $\mathrm{M} 2 \mathrm{M}$, which are essential for IoT. Low-Power Wireless Area Networks (LPWANs) aim to respond to such requirements. In fact, in order to achieve a low-power long-range communication, high receiver sensitivity is needed, as fine as to $-130 \mathrm{dBm}$ against -90 to $-110 \mathrm{dBm}$ in traditional technologies. This means that a higher energy per bit is needed, and thus that the modulation rate should be slower.

One of the major issues for M2M communications is the low-power long-range communication. LPWAN technologies, in order to achieve a long range capability, need to use high receiver sensitivities, up to $-130 \mathrm{dBm}$ compared with the -90 to $-110 \mathrm{dBm}$ in many traditional wireless technologies. This implies a higher energy per bit and thus a slower modulation rate. The two most popular solution for LPWAN are Sigfox and LoRaWAN. The first one is a global
IoT network, aiming a low deployment cost of long-range, small-message device communication. It is a narrowband technology using extremely slow Binary Phase Shift Keying (BPSK) modulation. The second one is an LPWAN specification for long-range low-datarate wireless battery operated devices communication, promoted by LoRa Alliance, which coverage is comparable to a cellular network.

3GPP addressed categories and new standards for IoT requirements [51]. New users categories (cat1-6) are introduced starting from LTE Release 8. Release 10 defines 6-8 categories, and Release 12 defines LTE Cat-0. This category suits IoT applications and general M2M applications needs with low data rates in short bursts. This results in a reduced complexity and power. NarrowBand-IoT (NBIoT) and LTE-M (or eMTC for enhanced Machine-Type Communications), are two complementary technoogies for IoT applications. NB-IoT is a direct candidate of Sigfox and LoRa, as it is less energy consuming and can address a large amount of connected devices. It can be deployed as a standalone solution or inside an LTE carrier, and will have more in-band deployment flexibility in 5G NR. LTE-M addresses another connected devices category which is more constrained in latency, mobility and density. Application examples are e-health, security, object tracking, etc. LTE-M however is more energy consuming. An illustration of LTE IoT evolution is given in Figure 10

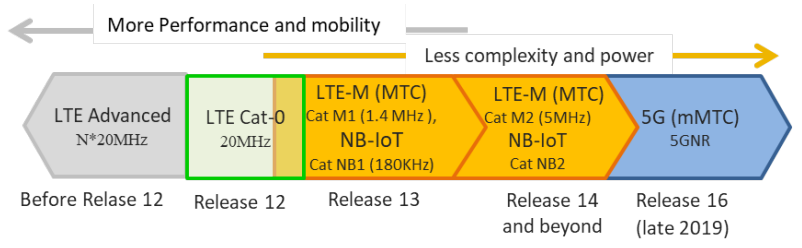

Figure 10: 3GPP IoT solutions standards

Note that many $5 \mathrm{G}$ technologies are specified to support IoT deployment, such as include D2D, SDN, NFV and MEC. For example, C-RAN reduces the cost of ultra-dense networks, expected especially with IoT deployments, through the simplification of BSs. This enables flexible and adaptable deployment.

\section{5G Energy considerations}

Today, energy is a major issue in all industries, including telecommunications. For example, the Mobile and wireless communications Enablers for Twenty-twenty Information Society (METIS) project considers EE as an important 5G topic [52]. The objectives of the project lead to technical goals with in particular a much denser network, containing low-power devices requiring higher battery life, up to 10 times longer than current batteries.

\subsection{Energy efficiency on network side}

$5 \mathrm{G}$ will densify the network in order to increase the coverage and the capacity of the system. However, as power consumption is expected to increase at the same time, a challenging issue for $5 \mathrm{G}$ is the EE. For example, considering 
data centres in the network, the author in [53] examines the variety of clusters and clouds that implies the need for powersaving control mechanisms. He presents network and cluster resources control algorithms, as well as an integrated and hierarchical control of the resources. Similarly, as treated by the project EARTH [54], improving EE at BS level need a variety of technologies. For example, it is proposed in [55] to use Cellular Partition Zooming (CPZ) to reduce energy consumption; following the present users in the area, the BS zooms in for coverage or out for sleep mode, which is energy saving.

Optimizations, categorized on green energy researches, aim BS energy use reduction [56]. In fact, the power consumption in the Operation Expenditure (OPEX) in today's mobile systems is dominated by networks, up to $90 \%$, and only $10 \%$ is for mobile user. The network electricity supply is thus a critical issue in mobile industry, especially for regions with difficult grid power connection. Alternative energy sources, light, wind, vibrations, etc. are considered. However, alternative energies, especially renewable ones, present some challenges regarding availability and volumes. In particular, combining renewable energy sources at BS is critical. For example, the japanese operator NTT DoCoMo launched in July 2004 the DoCoMo Eco Tower [57], which is an experimental an self-powered 3G BS. Thid BS uses solar and wind power at once. Similarly, Alcatel proposed Ecosustainable Wireless Networks to replace current generators [58]; Alcatel has an Alternative Energy Program combining alternative energy sources and energy-efficient equipments and networks.

Note that RF-EH appears as a candidate technology to further optimize energy management in 5G. In particular, EH from RF signals is actively studied and mathematical models are made in order to make it possible in the near future, as proposed in [59]. In the same scope, researches are interested in several scenarios, as cooperative multicast communication in [60]; EH relays harvest energy from the BS transmissions following two power splitting strategies at the relays for $\mathrm{EH}$, and analytical expressions of outage probability are given for these strategies.

\subsection{Energy efficiency on terminals side}

Battery life is a major constraint for equipments' design. In fact, it can be lengthened through enhancement of its capacity, use and recharge process. The overall EE of the network would be consequently improved. 5GPPP establishes requirements for future $5 \mathrm{G}$ systems considering ultra-efficient $5 \mathrm{G}$ hardware, especially in terms of energy consumption, flexibility and compatibility with heterogeneous environments [61]. The interface between network and terminal can use massive MIMO technique to enhance EE, in addition to new waveforms and to network radio resources optimization. The air interface, procedures, and signaling can also be enhanced in terms of energy consumption through sleep modes, simplified procedures access schemes, and more efficient modulation, spreading and coding that allow low transmission powers. Energy consumption can be reduced using EH from environmental energy sources, such as light, heat and vibration [62]. However, as they vary along location, time, weather conditions, etc., it can be difficult to guarantee a given Quality of Service (QoS) that is required in some wireless applications. A possible solution for this problem is ambient radio signals harvesting, i.e. RF-powered energyharvesting network (RF-EHN) [63, 64].

Note that circuit design has also to be coherent with EE optimization. Many technologies are discussed to prepare 5G-ready handsets, as in [65]. The research areas for that purpose include RF design, mobile cooperation, context awareness and seamless roaming among multiple technologies. New techniques for power amplification, antenna and filter design, and RF architecture are being studied for future handsets design. For example, M2M and D2D communications may use wireless power transfer technologies and optimization of sleep mode switching in order to have a battery-less sensor operation.

\section{RF Energy harvesting implementa- tion}

\subsection{Energy harvesting sources}

EH has been already widely deployed using several energy sources. It consists on absorbing ambient energy in order to utilize it instantly or later. The most popular harvested energy sources are solar energy, wind energy, tidal waves energy, mechanical energy, electromagnetic energy, thermal energy, etc. EH sources and their corresponding sensors are illustrated in Figure. 11][10].

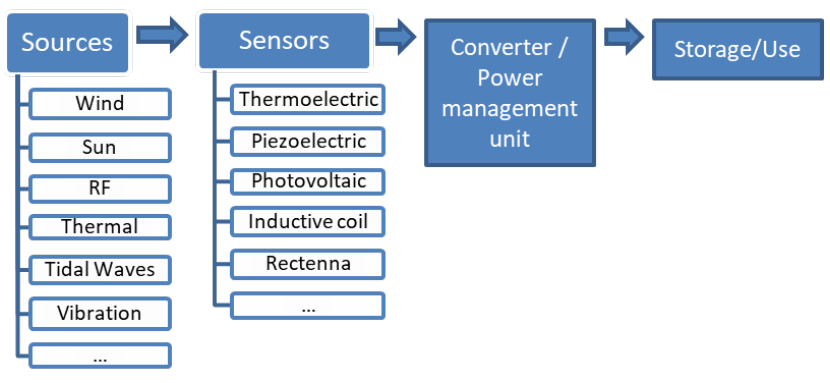

Figure 11: Energy harvesting sources and sensors

- RF sources have a power density ranging from 0.1 $\mu \mathrm{W} / \mathrm{cm}^{2}$ for ambient RF to $1000 \mu \mathrm{W} / \mathrm{cm}^{2}$ for dedicated RF [10]. Electricity in that case is produced through magnetic inductive coupling: a loop with time varying current induces a receive loop with an open circuit voltage, and the resulting voltage can thus be directly used or stored in a battery for later use.

- Harvesting energy from heat is based on thermal energy generator, which uses the difference of temperatures to generate electricity. However, it has a low efficiency $(5-10 \%)$ [50] and needs a temperature difference of $30^{\circ} \mathrm{C}$. The power density in this case is about $135 \mathrm{~mW} / \mathrm{cm}^{2}$ at $10^{\circ} \mathrm{C}$ [66].

- Sun and light EH, i.e. solar and photovoltaic energy, is very popular nowadays due to its power density, efficiency, and flexibility. The light is collected by a semiconductor material, usually silicon, that produces DC power by the movement of electrons in the 
semiconductor. The electricity is then stored in batteries or supercapacitors. The power density is about $100 \mathrm{~mW} / \mathrm{cm}^{3}$, but it requires direct exposure to light, which limits its use indoor [66].

- Mechanical energy is by the other hand harvested from vibration, pressure, etc. Among others, piezoelectric material, when exposed to a mechanical force, makes a polarization of ions in the crystal. This has a high energy density, which is about $50 \mu \mathrm{J} / \mathrm{N}$ [66]. An example of piezoelectric EH for IoT applications can be found in [50].

\subsection{Energy Harvesting constraints}

$\mathrm{EH}$ presents an interesting solution to current energy consumption issues. However, it also presents some design constraints, as ambient RF waves figure among the most challenging energy sources. It suffers more particularly from

- Causality, so that when harvested in the present, the energy is only available in the future, .

- Difficulty of concurrent EH and information transmission, at least for current designs.

- Efficiency loss when converting RF harvested signal to Direct Current (DC).

- Finite nature of energy storage.

For some applications, difficult environment access, connection cost and mobility makes it difficult to charge sensors or, at least, with a certain periodicity. In addition, if $\mathrm{EH}$ is used, for some EH sources, harvested energy depends on time and/or location and may not be enough for terminal's needs. Examples of time dependance are stillness time for vibration energy and night time for solar energy. Electromagnetic Radiation appears thus as an interesting stable and predictable energy source.

A cellular system can integrate EH using a standalone technique, hybrid technique or Simultaneous Wireless Information and Power Transfer (SWIPT) technique [67]. For the first technique, it allows to equip only some entities with EH. Hybrid EH empowers the system by both grid and EH, which may solve occasional unavailability of EH. SWIPT empowers the devices with harvested radio wave frequencies through which data is being received, as developed in 4.4.1.

Most of current mobile systems broadcast RF signals, carrying energy that presents a true opportunity as a source. At the same time, considering this possibility, the RF energy can supply devices. This implies the use of Wireless Power Transfer (WPT), and promising techniques are studied in order to implement such systems. The most significant examples are :

- Magnetic resonance coupling, which uses near-field signals and covers an extent ranging from some centimeters to some meters. This makes this technique adapted for charging mobile phones and hybrid electric vehicles, and its efficiency is $30 \%$ to $90 \%$ for a Tx-Rx distance ranging from $0.75 \mathrm{~m}$ to $2.25 \mathrm{~m}$ [68].
- Resonant inductive coupling, which exploits nearfield signals and can also be used suitable for mobile phones charging, passive RF IDentification (RFID) cards and contactless cards. Frequency bands for this technique range from $10 \mathrm{KHz}$ and $30 \mathrm{MHz}$ and have an efficiency from $6 \%$ to $90 \%$ respectively [69].

- RF energy transfer, which uses far-field waves. Its coverage ranges from few meters to several kilometers and is suitable for wireless body and WSNs. Its efficiency is $0.4 \%$ to over $50 \%$ for -40 and $-5 \mathrm{dBm}$ input power, respectively [70]. This technique is being increasingly researched these last years and is gaining growing maturity, which promises new networks concepts, such as Wireless Powered Communication Networks (WPCN) [71].

The circuit suggested in [72] for ambient RF energy battery charge, as may be used in IoT-like applications, is made of a rectifier circuit and an impedance matching network. It converts RF waves to Direct Current (DC). Unlike EH circuit, information decoding uses down-conversion and sampling to process the baseband signal. For EH, a dedicated circuit directly connected to a receiving antenna, called a rectenna (rectifier antennas), captures energy which is converted then into functional DC voltage. This operation is called RF-to-DC conversion.

A rectenna has five main components: an antenna, the network matching the output impedance of the antenna to the rest of the circuit, a low pass filter that eliminates higherorder harmonics, the rectifier which is a diode, and DC filter load (peak detector). Such rectennas are designed in [73], operating at $27.5 \mathrm{GHz}$, and achieve rectification with coupling between the input and output microstrip lines in the design. Note that EH-Oriented transceivers, especially rectennas, can be adopted in most cases for both indoor and outdoor applications and provide an efficient RF-to-DC conversion, reaching $80 \%$ in some configurations [74].

Figures 12 and 13 illustrate separately respectively energy and information receivers, which have to be combined for an EH scenario.

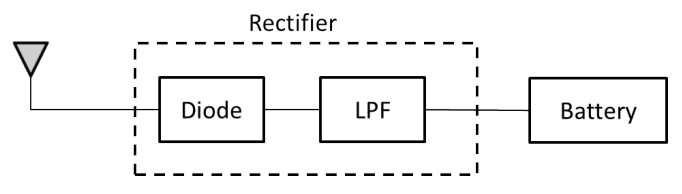

Figure 12: Simplified energy receiver scheme

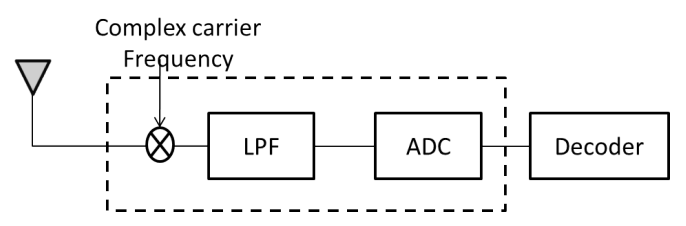

Figure 13: Simplified information receiver scheme

In the case of a receiver combining both $\mathrm{EH}$ and information decoding, the received power is split between the two either statically or dynamically. The splitting depends on the variations of RF energy and information, and also in the 
needs of the receiver. The combined receiver can separate information and energy circuits [75], as illustrated in Figure 14 , or it can integrate both in the same circuit, as illustrated in Figure 15.

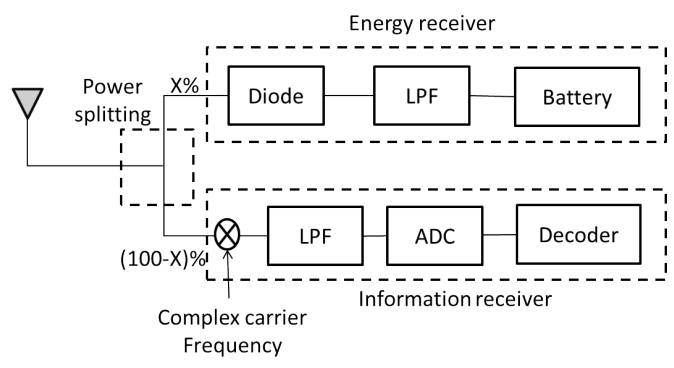

Figure 14: Separated information and energy receiver

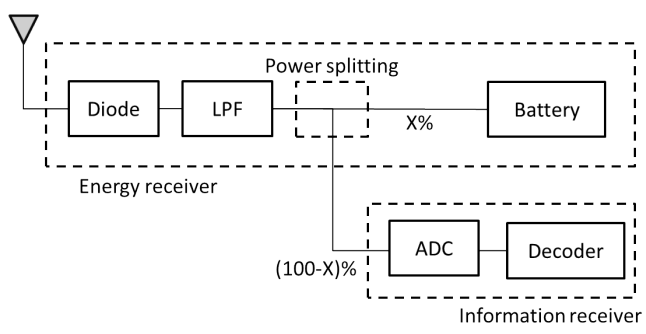

Figure 15: Integrated information and energy receiver

Authors in [75] show that there is a tradeoff exists between harvested energy and achievable rate, so that either integrated or separate receivers are selected depending on the harvested energy.

In teh remainder of this paper, RF-EH is considered. Note that RF energy by nature implies a relative time variability, as well as a sporadic aspect. On the other hand, this technology at mobile networks' scale is much younger than other energy sources harvesting, and accumulates thus less technology mastering and experience.

\subsection{RF Energy Harvesting hardware}

Several $5 \mathrm{G}$ use cases announce the need for self-sufficient and self-sustaining equipments. This energy chase covers the network as a whole including terminal devices, network elements and data centres, and $\mathrm{EH}$ can bring a solution for such requirements [72]. However, implementation of RFEH presents many challenges, including, in some scenarios, the conception of rectennas to collect RF energy and the WPT [73]. Two categories can be cited for WPT: generation of electric power within few meters, i.e. near-field, and generation in an extent that can reach few kilometers, i.e. far-field. The efficiency of energy transfer in near-field case can reach up to $80 \%$ [76]. The WPT in far-field appears as $\mathrm{RF}$ or microwave signal and uses antennas for transmission and reception then converts it to power by rectifier circuits.

From a hardware point of view, antenna designs already exist for EH from interference signals issued from GSM, WLAN, UMTS and related applications, where antennas are designed with the potential to be used in EH systems. Several categories are also proposed in the literature for different frequency bands such as $900 \mathrm{MHZ}, 940 \mathrm{MHz}, 1.95 \mathrm{GHz}$ and
2.44GHz, 0,8 to $12 \mathrm{GHz}$ [77, 78, 79, 80, 81]. The designed antennas can be integrated in an antenna array to increase the gain and preserve a very high frequency bandwidth.

Though the harvested energy remains suitable for low power electronics, as it is rather low compared to other EH sources. However, a major challenge for RF-EH is still the low power sensitivity and legal restrictions of maximum authorized RF power radiation.

$\mathrm{n}$ the other hand, following [82], the global EH market is expected to reach 974.4 Million USD in 2022 when it was only 268.6 Million USD in 2015. This growth is driven by electronic sensors with ultra low-power and by embedded systems, which involve an energy issue. EH can be used to solve this issue, as suggested in [83], and EH relays can be considered, especially when the devices use WPT through RF signals. These signals carry both information and energy, and suggest for relays to charge themselves by extracting energy from the signals they overhear.

Note that RF-EH chips are already being developed as indicated in [84], where a startup has raised 30 million USD in funding to develop chips that can be powered by ambient radio frequencies. More generally, EH from other sources is also coming to reality, as the market for EH chips will approach 3.4 billion USD by 2022 according to the report in [85]. The mentioned report cites some companies that are in the race for EH chip market, and include for example Apple, National Instruments, Analog Devices, STMicroelectronics, Powercast, Renesas, Microchip Technology, Texas Instruments, Mentor Graphics, etc. An example of optimizations for RF Energy in a WSN using Powercast can be found in [86].

\subsection{RF Energy harvesting approaches}

In order to supply power to the nodes through WPT, it is possible to use either BSs already present in the network, or dedicated Power Beacons (PBs) specifically deployed, as illustrated in Figures 16 and 17 The latter has the advantage of being easy to deploy as it doesn't need backhauling. In both cases, energy beamforming can improve the EH efficiency, and distributed cooperative beamforming can be used to provide data rate fairness [87].

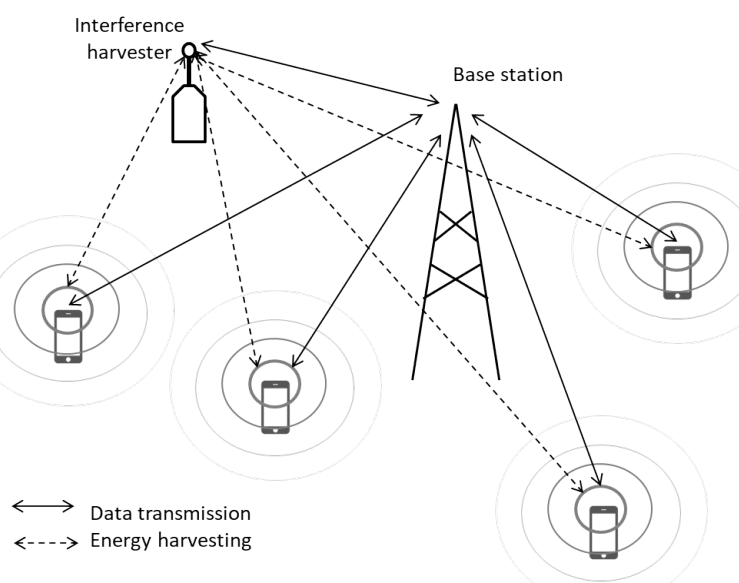

Figure 16: Illustration of the dedicated energy sources to supply RF energy 


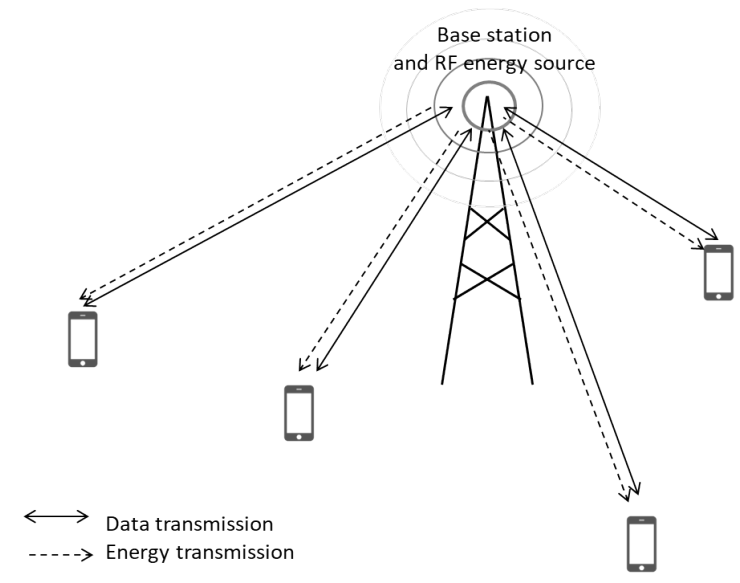

Figure 17: Illustration of the use of existing BSs to supply RF energy

\subsubsection{Simultaneous Wireless Information and Power Transfer}

Many suggestions for EH receiver architectures are inspired from the use of WPT in wireless networks. Actually, the same electromagnetic field can be used both for information and energy transfer to the end users and/or devices. This case is called Simultaneous Wireless Information and Power Transfer (SWIPT).

Two different tasks have to be completed by an EH receiver: decoding information and harvesting energy. The ideal case would integrate in the same receiver both functions with no power loss. However, realistic case dedicate a circuit for each task, so that $\mathrm{EH}$ and information decoding are performed separately. Authors in [75] suggest an intermediate solution that partially combines EH and information decoding circuits in a manner that the rectifier performs a part of the information decoding.

In a SWIPT configuration, a time slot, or a received signal power portion, are used for EH. The rest of time, or of power, are intended for decoding information. Nevertheless, time sharing scheme is more interesting as energy receivers and information receivers, which are usually distinct, have different power sensitivities. Note that there is a tradeoff between energy transfer rate and information transmission rate following the channel. For example, in an Additive White Gaussian Noise (AWGN) channel, maximum information rate target and maximum power transfer efficiency target are compatible.

There are technological issues that still need to be addressed in order to enable SWIPT use for wireless network deployment. For example, if we consider the nature of the transfers in SWIPT, the variation detection in RF signal by the receiver defines the information amount it can decode. The relationship between the two can be described by the Signal to Interference plus Noise Ratio (SINR), i.e. ratio between the power of the signal power and the disturbance power, including interference, noise and eventually any other disturbance affecting the decoding. In practice, even though a signal power can be low, its information rate could be very high. This is however not true for energy amount carried by an RF signal, which depends on its magnitude only. Hence, in a SWIPT, there is a trade-off between transferred energy amount and information rate, as it is not possible to maximize the "rate" of the two transfers simultaneously. Consequently, there is a need to adapt te network design for SWIPT implementation, leading to research issues on the design, analysis and optimization of SWIPT-based networks architectures and protocols. For example, in [88], the wireless powering of terminals in an UDN considers game theoretical approach of power allocation in a network of Full-Duplex (FD) BSs which serve an area highly dense of half-duplex (HF) user equipments. Considering EE and coverage optimization problem for such network as a mean field game gives an optimal strategy to adapt BSs for this type of network settings. SWIPT can also be considered in cooperative communication networks, as proposed in [89], where relays harvest powers to be able to amplify and forward data. An optimization, such as maximum ratio combining, can be then operated considering direct and relayed signals.

Note that there are many variants of SWIPT schemes following the integration in the model of other $5 \mathrm{G}$ techniques or the consideration of specific use case. An example is given in [87], where a cooperative communication network is considered with a distributed energy beamforming and simultaneous communication with several users. Moreover, when used with NOMA, efficient EH can be performed, and EE can be enhanced with the use of EH pilots for channel estimation.

\subsubsection{Dedicated RF sources powering}

Many tools are proposed in case of powering through dedicated RF energy source, as in [90] where optimal power allocation is suggested for frequency division multiplexing, meaning that data transmission and $\mathrm{EH}$ can be performed simultaneously, and time division multiplexing, meaning that data transmission and $\mathrm{EH}$ are implemented in different time slots but in the same frequency band. Optimal EH and information transmission strategy is then given and long-term throughput can be optimized. Note that authors in [91] considered the possible optimizations in the case of dedicated energy sources for WPT as in IoT applications. Authors showed that power control is critical for system achievable rate improvement in the case of a finite blocklength, and when the Tx power is asymptotically optimum, performance is nearly optimum.

PBs provided by the network, can be integrated in a relay network. For example, [92] suggests that relay nodes harvest transmitted power from the PB station to forward signals to destination. The information can be relayed but also power, following adequate protocols to optimize power splitting, especially in the presence of multi-antennas. Another example using dedicated RF charging in the case of smart wearables, where the energy consumption is very low, is discussed in [93]. This implies a mix of operator-deployed and user-deployed systems that will need to cooperate. Authors in fact highlight through system-level evaluation the preference for omnidirectional energy transmission in this case.

\subsubsection{Interference harvesting}

An increasing number of users are actively using mobile systems and demanding continuously improved quality of 
experience. In addition to the diversity of end devices and of applications, each with its own constraint, 5G uses a heterogeneous UDN to respond to the current landscape, which already implies the coexistence of many RF signals on different bands. In fact, as illustrated in Figure 18 , today's current deployments imply the presence of many signals, which can be harvested to power devices. In particular, GSM bands present a higher RF power density, while other sources may also be exploited for energy harvesting, such as Amplitude Modulation (AM) radio stations and Ultra High Frequency (UHF) RFID.

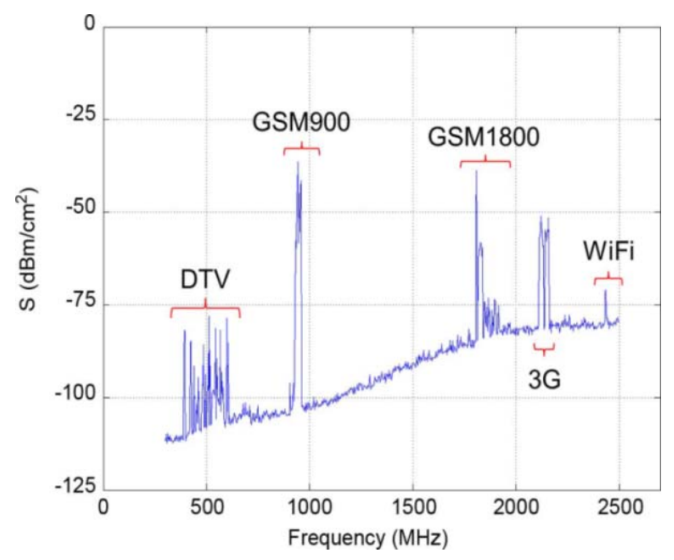

Figure 18: Example of measured RF power densities of main RF energy sources in an urban outdoor location (Source [94])

A given device using a given system is exposed to signals intended for other users in the same network, signal from other networks, or signals on other bands. These signals are usually seen as interference and many techniques and resources are used to mitigate it. It has to be carefully dealt with in an EH context for different types of channels, including broadcast channels, multicast channels, multiple access channels, and multi-user interference channels, as reviewed in [95]. However, they can also be seen as an energy source to harvest.

Figure 19 illustrates the case where signal from the user can be harvested by an interference harvester, which is a node capable of communicating itself with the BS. The harvesting and transmitting are usually sequential.

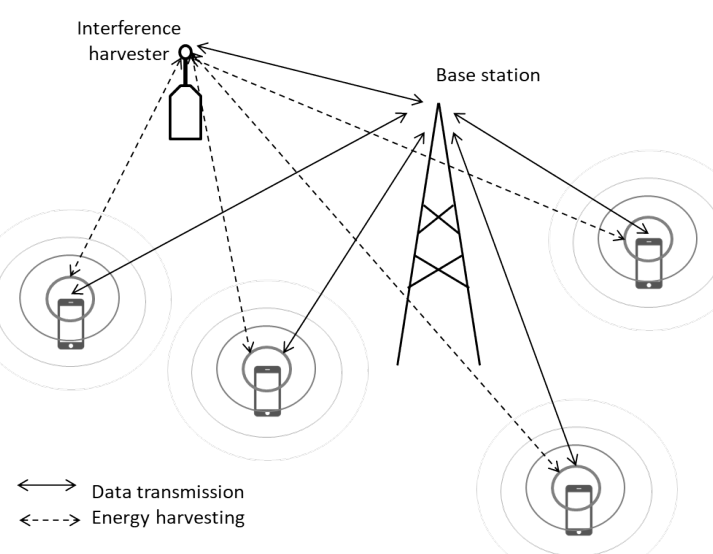

Figure 19: Example of an interference Harvesting scenario

The authors in [96] present opportunistic communication for devices that can reconfigure according to the the band they use, which makes them able to separate useful signal from possibly harvestable OOB interference. Nevertheless, interference harvesting from the same band requires additional processing, and strategies to realize this harvesting are currently being researched, and promising to be a key enabling factor for self-sustainable transmissions in 5G networks [97]. Similarly, authors in [98] present a cooperative wirelessly powered communication network protocol, where distant users overhear uplink signals of users and downlink access point broadcast signals, and time allocation for uplink and downlink are jointly optimized, which increases users rate and improves user fairness and transmission delay. Actually, the analysis of RF-EH performance has been considered in works such as [99], where the harvested energy mean, transmission outage probability and power outage probability are studied.

The energy can be also harvested from parts of the signal of the same user. An example with multicarrier modulations, as in [74], considers the fact that the Cyclic Prefix (CP) is used when still analog and thus, its energy can be harvested. The CP would remove inter-symbol interference, and at the same time contribute in SWIPT implementation, especially for WPT in downlink.

A backscatter communication architecture is suggested in [100], which combines ambient EH and reflection of this same energy modulated, with all its related signal processing aspects and multiple access techniques.

\subsection{RF Energy harvesting scenarios}

Globally, as enumerated in [95], three EH and transmission protocols can be identified:

- Harvest-Use (HU), for which the device is powered by EH directly, without using any buffer. Data transmission is thus possible only when harvested energy can cover the processing energy cost.

- Harvest-Store-Use (HSU), for which a storage device, typically a battery, is used to gather harvested energy. This energy is then available for use only in the next time slot. This case has to consider carefully possible storage inefficiency during the charging and leakage during the storage. For example, only about $70 \%$ of the energy is stored in Ni-MH rechargeable batteries while $95 \%$ can be reached with a supercapacitor. However, a battery implies less leakage than a supercapacitor.

- Harvest-Use-Store (HUS), which uses two energy storage devices : a supercapacitor storing the harvested energy for immediate use, and a buffer storing the remaining energy for later use.

The energy buffer is critical when using one of these protocols. Other schemes in a different optimization perspective can consider storage constraints, QoS requirements or capacity achieving targets. An example is the introduction of a sleep-and-awake mechanism to enhance the achievable rate [95]. 


\subsection{RF Energy harvesting scheduling}

In order to have an efficient EH system, and depending on the used EH protocol used, the energy consumption has to be adapted to the EH. This is to prevent energy outage or overflow. Thus, the design of energy use scheduling is critical. It can be offline or online, following the case where channel state information is available at the beginning of the transmission or only on the fly.

Energy scheduling depends on many parameters, such as data throughput, transmission competition time, mean delay, outage probability, quality of coverage, message importance, grid power consumption when there is a hybrid power supply, etc. For instance, the scheduling of RF harvested energy optimizes the time duration of information processing and EH. A tradeoff between the minimum information outage probability and the maximum average harvested energy defines the optimal mode switching between the two functions at the receiver. A review of scheduling techniques can be found in [95].

The signaling in wirelessly empowered communication networks has also to be carefully designed. In fact, if wireless powered communication networks control is centralized, signaling overhead, as well as optimizations in case of large number of devices can be prohibitively complex. Thus, a distributed EH random access protocol can be adopted, as in [101], where a slotted ALOHA is used a harvest-until-access protocol. The proposed algorithm optimizes the average channel throughput without knowledge of full channel state information, especially when the number of devices is large, which reduces the complexity and overhead of a centralized management.

Integrated spectrum and energy control mechanisms are presented for a typical heterogeneous 5G network in [102]. In this work, the authors consider the network where the devices have different EH capabilities and perform a cooperative resource sensing for CR implementation. In this case, both energy and spectrum are "harvested". This leads to an architecture and model with specific spectrum and energy control mechanisms in many 5G scenarios, and consequently a Medium Access Control (MAC) protocol for this context. Another energy and spectrum efficiency optimized MAC protocol for 5G EH can be found in [103], where SWIPT is considered for cooperative and non-cooperative schemes with $\mathrm{EH}$ capabilities in a CR aided $5 \mathrm{G}$ networks.

\section{RF Energy harvesting in $5 \mathrm{G}$ ecosys- tem}

$5 \mathrm{G}$ ecosystem integrates a wide range of use cases, using established and new technologies. $\mathrm{EH}$ is researched to be integrated with key $5 \mathrm{G}$ enablers, and examples of these researches are given in the remainder of this section.

\subsection{UDN RF-EH-enabled}

UDN are among main 5G enablers for many use cases, including IoT. Considering ambient RF-EH in dense networks, many implementation and operation challenges arise including energy availability, EH mode selection, cooperation be- tween BSs and devices. In particular, there are tradeoffs between EE, SINR and outage probability, as highlighted in [104], where it is found that co-channel interference EH can bring efficiency improvement, especially if combined with an optimization of BSs parameters.

When the capacity is limited, the $\mathrm{H} 2020$ project SCAVENGE [105] suggests that cells can be split so that some are master Access Nodes (AN) while the others are EH AN. In case of heavy traffic, all ANs would be enabled to provide required capacity, while for light traffic, a part of EH AN can be switched off. This can be possible as UDN have high probability of Line-of-Signt, making the access easier for devices without the need of EH ANs. Backhauling the cells, if using wireless connection, makes the deployment cheaper and more flexible. In particular, EH ANs backhauling can use mmWave or be self-backhauling. In the second case, the access and the backhauling use the same link, shared in time and/or frequency. In the first case, mmWave can provide EH capabilities for rapid AN installation. In particular, for some deployment scenarios, micro cells with low power can be used, for which an EH powering and a wireless backhaul are suitable. The use of EH in this case, as indicated in [105], can be cost-effective. It can also provide an easy coverage and deployment in rural areas, easy cell planning, reduced environmental impact and cost saving in long-term.

Note that WPT can be used between BSs in addition to terminals. This will imply a new cell planning based for example on energy balancing connection and QoS-aware BS planning.

\subsection{RF-EH in C-RAN}

C-RAN and H-CRAN aim to provide a high data rate, energy efficient network. However, because of its heterogeneous nature, H-CRAN is more energy consuming as it has a larger number of RRHs than C-RAN. When the network is dense, the energy requirements become critical and several adaptations and optimizations have to be made. EH can bring thus a solution as in [106], where BSs are suggested to be powered with $\mathrm{EH}$, and can also power devices. A throughput optimization in that case can be performed under receiver charged energy constraint. The grid energy consumption in the case H-CRAN downlink with one grid-powered BS and many RRHs EH-powered is studied in [67] and a model and optimization of user association, power allocation and EH constraints are given. In this scenario, the EE optimization is modeled as a fractional mixed integer nonlinear programming problem, which can be solved in few iterations. This improves the number of users, who are mostly associated to EH-RRHs, and thus decreasing the grid energy consumption.

In a UDN deployment, the implementation cost can be high due to infrastructure and small cells backhauling, and C-RAN can en be used to reduce this cost, especially for low data rate traffic as in IoT. The fronthauling then can use EH for an energy efficient deployment [105]. Similarly, the energy cost in an RF-EH combined with an energy management and scheduling in H-CRAN context is considered in [107]. RRHs in that case are EH-powered, and a hierarchical framework based on economical model and using energy sharing and energy trading between $5 \mathrm{G}$ actors, and more specifically internet service providers, can provide a 
framework for the system design.

\subsection{RF-EH enabled MEC}

In MEC, computing resources are available at the edge of the mobile network, i.e. BSs, in order to ensure low latency and to reduce core network traffic. Globally, two main scenarios are considered for $\mathrm{EH}$ and MEC combination. Either EH capability is deployed at the MEC BS (or resource server) to prevent possible grid energy absence or unavailability in some scenarios, like natural disasters or remote areas, or it is implemented on device side, which is more suitable in case, for example, of IoT. Study of the state of the art for these two scenarios can be found in [108]. For the first scenario, energy forecast and $\mathrm{EH}$ enabled BSs load balancing have to be controlled. An example considers BSs equipped with EH capabilities and may reduce thus by the same occasion energy consumption. The authors in [109] suggest to optimize the BSs and virtual MEC resources use following traffic load and forecasted harvested energy parameters. The heuristic optimization employs sleep mode for BS and softscaling for Virtual machines. The authors announce up to $69 \%$ energy saving in single BS case, that can be enhanced by up to $16 \%$ in BSs cluster. Similarly, an illustration of the second scenario can be found in [110] and present a low-complexity dynamic computing offloading strategy for MEC with EH. This strategy decides computation offloading power and device transmit power, without the need of distributed information, and is easily implementable, besides being asymptotically optimum.

\subsection{D2D with EH}

D2D communication suffers from a major constraint, which is energy. EH can be thus integrated to devices in order to solve this issue, and many studies consider this case and provide theoretical performance derivation for different scenario. For example, in [111], the authors consider the scenario where UEs in a traditional cellular network assisted with relays use decode-and-forward cognitive D2D communication and $\mathrm{EH}$. They provide analytical expression for main performance parameters, which helps to optimize link robustness. Another scenario can consider D2D in large-scale cognitive cellular networks as by authors in [112], where D2D transmitters harvest energy from dedicated multi-antenna PBs. A power transfer model with three possible power transfer policies can be proposed, in addition to an information signal model with two considered receiver selection cases. In addition to outage probability, secrecy is also considered in the analysis conducted by the authors and complete the framework for secure D2D communication.

\subsection{RF-EH with key 5G RAN techniques}

Spectral efficiency is critical in current mobile systems, particularly in RAN. Thus, spectrum optimizations have to be considered, in combination with RF-EH techniques. Reviewed techniques in the rest of this section include cooperative communications and cognitive radios, in addition to massive MIMO, NOMA and D2D. The combinations and optimizations usually consider many of these techniques at a time, in order to prepare its integration on practical systems.

\subsubsection{Cooperative communications}

In an EH network, nodes may have different channel and energy conditions, which may enhance performance using a cooperative approach. Transmission schemes for cooperative networks, multi-user networks, cognitive radio networks and cellular networks are thus to be considered.

Multi-user environment is an example of scenarios with $\mathrm{EH}$, where interference management mechanisms have to be effective. Moreover, this environment presents spatial diversity gains that offer an opportunity to enhance system performances through collaboration between network nodes, which form a virtual MIMO system. Among studied aspects for cooperative networks, the power allocation is to be optimized under EH models, as well as throughput in delay constrained environment, and joint power allocation and time scheduling with EH.

Another example of EH integration issues is the joint optimizations of EE and frequency bands in the design of wireless-empowered transceivers, as presented in [74] where in-band interference harvesting is designed so to realize selfsustainable communication architecture.

\subsubsection{NOMA}

The adaptation of EH with $5 \mathrm{G}$ coming techniques, such as NOMA or CR networks, is an important issue to address for RF-EH deployment. In fact, there is a need for more comprehensive design for both new technologies, NOMA and RF-EH. Such combination may if established provide an efficient use of the energy and radio spectrum in energy efficient and sufficient networks, as in [113]. Other examples of issues are modeling and analysis of large scale EH networks and relay-based EH networks, as well as the design of energy cooperative strategies between harvesting devices. Note that in addition to energy and power transfers, it is fundamental to consider energy storage at end nodes, which ideally would consume harvested energy immediately within a transmission cycle.

Analysis tools for EH NOMA are intensively investigated, as in [114] where users outage probability under imperfect channel state information and imperfect SIC in a cooperative EH NOMA network is derived. Another example of mathematical modeling for user grouping, power and time allocation can be found in [115], which adopts a mesh adaptive direct search algorithm to optimize data rate and transmit power for each user with reasonable complexity. Similarly, outage probability is calculated for relay systems with NOMA and EH in [116], where the multiuser downlink is considered and BSs use multiple antennas to beamform information and energy.

NOMA use in specific wireless powered IoT relay systems with RF-EH capabilities can be found in [117], where RF-EH and information transmission system with time switching and power splitting relaying are considered. Due to the constrained nature of IoT nodes, the authors propose for IoT nodes to first harvest energy from the source node, either with time switching relaying or power splitting relaying 
protocol. After that, the information of the source node is transmitted by the relay node as well as its own information using NOMA. The description of this system is mathematically derived by the authors, allowing several optimizations of system parameters.

\subsubsection{Massive MIMO}

Massive MIMO is a key $5 \mathrm{G}$ enabler, and its integration with EH schemes has been studied by many researchers. In [118], the authors explore the use of massive MIMO in heterogeneous networks. The deployment considers macrocells where users harvest energy to maximize their uplink power by minimizing uplink pathloss. It is found that user association should be optimized to maximize both harvested energy and information transfer. In this case, the downlink received power and the uplink received power should be both considered in the optimization. The stochastic model for the harvested energy and its link to uplink achievable performance demonstrates the added value of massive MIMO on that point, though the gain may deteriorate with network density. The proposed model enables however the study of massive MIMO and RF-EH integration with other technologies.

Another example of $5 \mathrm{G}$ techniques integration can be found in [119], where the downlink of a C-RAN enabled scenario with distributed large-scale MIMO is considered. Each $\mathrm{RRH}$ is powered $\mathrm{EH}$ and the grid in order to secure the RRHs from possible EH unavailability. The problem problem of BS energy consumption minimization under QoS constraints in this scenario can be solved using linear programming or an online energy management algorithm.

SWIPT in massive MIMO systems is studied in [120], where an analytical study of hybrid and full-dimensional processing is performed. It considers hybrid precoding and beamforming BS and derives expressions for harvested energy, achievable rates and energy-rate tradeoff for growing number of BS antennas and network nodes. A hybrid precoding and combining is proposed which optimizes harvested energy with little energy-rate tradeoff degradation.

\subsection{4 mmWave}

mmWave is attractive for $\mathrm{EH}$ as it is intended to be integrated for large antenna arrays and a dense network, presenting thus interesting harvesting opportunities. However, it propagation characteristics, i.e. poor penetration and diffraction, may limit its use to some scenarios. Many studies though consider RF-EH for mmWave communications. For example, authors in [121] consider a scenario where mmWave signal provides energy and/or information to low power devices and derive key information and $\mathrm{EH}$ performance parameters as well as overall coverage performance. This establishes an analytical framework to design network and device parameters, for example for antenna geometry optimization.

A study on combination of NOMA with massive MIMO using mmWave can be found in [122], where SWIPT is used to enhance EE. Hybrid precoding is used for mmWave massive MIMO, and information and energy can both be "harvested" by users thanks to a power splitting receiver. Algorithms are proposed to select a user per beam, and an analog precoding is then performed to all beams, then users grouping is performed and a digital precoding is applied. An iterative joint optimization of power allocation and power splitting reaches finally a maximized achievable sum-rate.

Note that antennas design for mmWave specific applications are also a challenge, as investigated in [123], where the authors consider ambient RF-EH antenna on textile for wearable in mmWave, and provides up to $40 \%$ on-body and $60 \%$ off-body radiation efficiency.

\subsubsection{Cognitive Radio}

Cognitive radio environment needs to be adapted with EH. As already mentionned, CR is often considered with other $5 \mathrm{G}$ enabling technologies. In fact, the spectrum usage is optimized as Secondary Users (SUs) use the spectrum of Primary users (PUs), following different priorities and cooperation strategies. These strategies may incorporate information transmission and spectrum sharing among nodes, but also EH strategies. In this case, both PU and SU get benefits as the PU improves his throughput through SU as relay node, and SU gets more spectrum usage opportunities from PU's spectrum. In addition, PU's bands access can be supported by SU's harvested energy. Conventional cognitive radio networks, e.g., spectrum allocation and management, spectrum sensing and handover, spectrum sharing, are reconsidered to enhance the network reliability in an $\mathrm{EH}$ context where, in addition, harvesting, spending or conserving energy have to be optimized.

An example of transmission CR schemes in RF-EH network can be found in [124], where an analytical study characterizes dedicated PBs. Information transfer and EH operate asynchronously in different bands. Another example considers a two-way relay assisted CR NOMA network [125]. In this example, a joint optimization of power allocation, transmit power and power splitting ratio is proposed. Similar throughput maximizations under QoS constraints can be found in [126] for a CR context.

\section{Conclusion}

This paper is a reflection of the increasing interest on new techniques to make energy use more efficient in 5G systems. It aims to provide a comprehensive review on RFEH-enabled $5 \mathrm{G}$ networks. To this end, 5G standardization, requirements, use cases, ecosystem and key enabling technologies were reviewed, and particularly their energy aspects. RF-EH was then highlighted as a promising technique to solve energy supply and/or optimization. Different aspects of EH were exposed, including implementation and network protocols. The paper then presents literature review of RF$\mathrm{EH}$ integration with $5 \mathrm{G}$ enabling technologies, as well as some research trends and challenges following the integrated technologies.

Conflict of Interest The authors declare no conflict of interest. 


\section{References}

[1] S. El Hassani, H. El Hassani, and N. Boutammachte, "Rf energy harvesting for 5g: An overview," in 2017 International Renewable and Sustainable Energy Conference (IRSEC), Dec 2017, pp. 1-6.

[2] Cisco, "Cisco, visual networking index: Global mobile data traffic forecast ,update 2013-2018," Tech. Rep., 2014.

[3] "5g energy performance, ericsson white paper," Ericsson, Tech. Rep., apr 2015.

[4] "Driving the convergence of the physical and digital world - white paper on next generation mobile technology," ZTE Corp, Tech. Rep., 2014.

[5] "Sktelecom 5g white paper (white paper,version 1.0)," SKTelecom, Tech. Rep., oct 2014.

[6] S. Buzzi, C. I, T. E. Klein, H. V. Poor, C. Yang, and A. Zappone, "A survey of energy-efficient techniques for $5 \mathrm{~g}$ networks and challenges ahead," IEEE Journal on Selected Areas in Communications, vol. 34, no. 4, pp. 697-709, April 2016.

[7] Y. He, X. Cheng, W. Peng, and G. Stuber, "A survey of energy harvesting communications: models and offline optimal policies," Communications Magazine, IEEE, vol. 53, no. 6, pp. 79-85, June 2015.

[8] I. B. Sofi and A. Gupta, "A survey on energy efficient $5 \mathrm{~g}$ green network with a planned multi-tier architecture," Journal of Network and Computer Applications, vol. 118, pp. $1-28$, 2018. [Online]. Available: http: //www.sciencedirect.com/science/article/pii/S1084804518302029

[9] T. Soyata, L. Copeland, and W. Heinzelman, "Rf energy harvesting for embedded systems: A survey of tradeoffs and methodology," IEEE Circuits and Systems Magazine, vol. 16, no. 1, pp. 22-57, Firstquarter 2016.

[10] S. Divakaran, D. D. Krishna, and N. Nasimuddin, "Rf energy harvesting systems: An overview and design issues," International Journal of RF and Microwave Computer-Aided Engineering, p. e21633, 12 2018.

[11] "3gpp commits to $5 \mathrm{~g} \mathrm{nr}$ in unlicensed spectrum in its next release," https://www.qualcomm.com/news/onq/2018/12/13/ 3gpp-commits-5g-nr-unlicensed-spectrum-its-next-release Press News, Tech. Rep., Dec 2018, accessed: 2019-07-26.

[12] "FP7 ICT project : Mobile and Wireless Communications Enablers for the Twenty-Twenty Information Society 5G,' https://www. metis2020.com/. Tech. Rep., 2012, accessed: 2019-05-11.

[13] "White paper - 5g use cases and requirements future works," Nokia Networks, Tech. Rep., 2014.

[14] “5G White Paper,” NGMN Alliance, Tech. Rep., 022015.

[15] "5g and energy," https://5g-ppp.eu/wp-content/uploads/2014/02/ 5G-PPP-White_Paper-on-Energy-Vertical-Sector.pdf 5 G PPP, Tech. Rep., 2015, accessed: 2017-12-16.

[16] M. S. C. A. H2020-MSCA-ITN-ETN, "Sustainable cellular network harvesting ambient energy," http://www.scavenge.eu/ Tech. Rep., accessed: 2019-07-28.

[17] "Follow the leaders on technology, tools, and techniques : Understanding and testing the $5 \mathrm{~g}$ ecosystem," http://literature.cdn. keysight.com/litweb/pdf/5992-3144EN.pdf Keysight TECHNOLOGIES, Tech. Rep., jul 2018, accessed: 2019-07-30.

[18] M. Medin and G. Louie, "The 5g ecosystem: Risks \& opportunities for dod," https://media.defense.gov/2019/Apr/03/2002109302/-1/-1/ 0/DIB_5G_STUDY_04.03.19.PDF . Defense Innovation Board, Tech. Rep., apr 2019, accessed: 2019-07-30.

[19] M. CAGENIUS, A. RYDE, J. VIKBERG, and P. WILLARS, "Simplifying the $5 \mathrm{~g}$ ecosystem by reducing architecture options," https://www.ericsson.com/48f8e1/assets/ local/publications/ericsson-technology-review/docs/2018/ etr-5g-core-radio-migration.pdf Ericsson Technology review, Tech. Rep., nov 2018, accessed: 2019-07-30.

[20] J. A. Manner, "Wrc-19 - an opportunity to bridge the digital divide for 5g: Opinion," https://news.itu.int/ wrc-19-opportunity-to-bridge-digital-divide-5g/ ITU News, Tech. Rep., May 2019.
[21] "Samsung announces world's first $5 \mathrm{~g}$ mmwave mobile technology," https://news.samsung.com/global/ samsung-announces-worlds-first-5g-mmwave-mobile-technology Samsung Corp., Tech. Rep., May 2013, accessed: 2019-07-26.

[22] "Huawei to bring $73 \mathrm{ghz}$ mmwave mu-mimo live demo to deutsche telekom," http://www.huawei.com/en/news/2016/2/ 73GHzmm-Wave-Mu-MIM-live-demo Huawei press, Tech. Rep., Feb 2016, accessed: 2019-07-26.

[23] A. Bleicher, "First intercontinental $5 \mathrm{~g}$ trial begins at winter olympics," http://www.huawei.com/en/news/2016/2/ 73GHzmm-Wave-Mu-MIM-live-demo. IEEE Spectrum, Tech. Rep., Feb 2018, accessed: 2019-07-26.

[24] X. Hong, J. Wang, C. Wang, and J. Shi, "Cognitive radio in 5g: a perspective on energy-spectral efficiency trade-off," IEEE Communications Magazine, vol. 52, no. 7, pp. 46-53, July 2014.

[25] E. Paulson, M. KAMALUDIN, S. Kamilah, and U. Dauda, "Cognitive radio in $5 \mathrm{~g}$ - a smart city perspective-," in The 2017 MalaysiaJapan Workshop on Radio Technology, 012017.

[26] R. Alkhansa, H. Artail, and D. M. Gutierrez-Estevez, "Lte-wifi carrier aggregation for future $5 \mathrm{~g}$ systems: A feasibility study and research challenges," Procedia Computer Science, vol. 34, no. 0, pp. 133 - 140, 2014, the 9th International Conference on Future Networks and Communications (FNC'14)/The 11th International Conference on Mobile Systems and Pervasive Computing (MobiSPC'14)/Affiliated Workshops. [Online]. Available: http: //www.sciencedirect.com/science/article/pii/S1877050914009235

[27] M. Alam and M. Ma, "Resource matching in carrier aggregation enabling 5g networks," Wireless Personal Communications, 102016.

[28] H. PAPADOPOULOS, C. Wang, O. BURSALIOGLU, X. Hou, and Y. Kishiyama, "Massive mimo technologies and challenges towards $5 \mathrm{~g}$," IEICE Transactions on Communications, vol. E99.B, pp. 602 $621,032016$.

[29] Z. Gao, L. Dai, D. Mi, Z. Wang, M. A. Imran, and M. Z. Shakir, "Mmwave massive-mimo-based wireless backhaul for the $5 \mathrm{~g}$ ultradense network," IEEE Wireless Communications, vol. 22, no. 5, pp. 13-21, October 2015.

[30] T. Rappaport, S. Sun, R. Mayzus, H. Zhao, Y. Azar, K. Wang, G. Wong, J. Schulz, M. Samimi, and F. Gutierrez, "Millimeter wave mobile communications for $5 \mathrm{~g}$ cellular: It will work!" Access, IEEE, vol. 1, pp. 335-349, 2013.

[31] "5g channel model for bands up to100 ghz," IEEEGC, dec 2015.

[32] X. Zhang, M. Jia, L. Chen, J. Ma, and J. Qiu, "Filtered-ofdm - enabler for flexible waveform in the 5th generation cellular networks," in 2015 IEEE Global Communications Conference (GLOBECOM), Dec 2015, pp. 1-6.

[33] A. Farhang, N. Marchetti, F. Figueiredo, and J. P. Miranda, "Massive mimo and waveform design for 5th generation wireless communication systems," in 1st International Conference on 5 G for Ubiquitous Connectivity, Nov 2014, pp. 70-75.

[34] F. Schaich, T. Wild, and Y. Chen, "Waveform contenders for 5g suitability for short packet and low latency transmissions," in 2014 IEEE 79th Vehicular Technology Conference (VTC Spring), May 2014, pp. 1-5.

[35] N. Michailow, M. Matthé, I. Gaspar, A. Navarro Caldevilla, L. Mendes, A. Festag, and G. Fettweis, "Generalized frequency division multiplexing for 5th generation cellular networks (invited paper)," IEEE Transactions on Communications, vol. PP, pp. 1-18, 082014.

[36] A. Benjebbour, K. Saito, A. Li, Y. Kishiyama, and T. Nakamura, "Non-orthogonal multiple access (noma): Concept, performance evaluation and experimental trials," in 2015 International Conference on Wireless Networks and Mobile Communications (WINCOM), Oct 2015, pp. 1-6.

[37] S. Timotheou and I. Krikidis, "Fairness for non-orthogonal multiple access in 5g systems," IEEE Signal Processing Letters, vol. 22, no. 10, pp. 1647-1651, Oct 2015.

[38] M. Al-Imari, P. Xiao, M. A. Imran, and R. Tafazolli, "Uplink non-orthogonal multiple access for $5 \mathrm{~g}$ wireless networks," in 2014 11th International Symposium on Wireless Communications Systems (ISWCS), Aug 2014, pp. 781-785. 
[39] M. Vaezi, Z. Ding, and H. V. Poor, Multiple Access Techniques for $5 G$ Wireless Networks and Beyond. Springer International Publishing, 2018.

[40] S. R. Islam, M. Zeng, and O. Dobre, "Noma in 5g systems: Exciting possibilities for enhancing spectral efficiency," 062017.

[41] M. Aldababsa, M. Toka, S. Gokceli, G. K. Kurt, and O. Kucur, "A Tutorial on Nonorthogonal Multiple Access for 5G and Beyond," arXiv e-prints, p. arXiv:1902.08992, Feb 2019.

[42] C. Mobile, "C-ran the road towards green ran (white paper,version 3.0)," Tech. Rep., dec 2013.

[43] J. Li, M. Peng, Y. Yu, and Z. Ding, "Energy-efficient joint congestion control and resource optimization in heterogeneous cloud radio access networks," CoRR, vol. abs/1602.05351, 2016. [Online]. Available: http://arxiv.org/abs/1602.05351

[44] G. T. . version 15.3.0 Release 15) Technical specification, "System architecture for the 5g system," https://www.etsi.org/deliver/etsi_ts/ 123500_123599/123501/15.03.00_60/ts_123501v150300p.pdf Tech. Rep., sep 2018, accessed: 2019-07-28.

[45] D. d3.1 : Functional network architecture and security requirements, "5g novel radio multiservice adaptive network architecture (5g norma),"'https://5gnorma.5g-ppp.eu/wp-content/uploads/2016/ 11/5g_norma_d3-1.pdf Tech. Rep., dec 2015, accessed: 2019-07-28.

[46] P. K. Agyapong, M. Iwamura, D. Staehle, W. Kiess, and A. Benjebbour, "Design considerations for a $5 \mathrm{~g}$ network architecture," IEEE Communications Magazine, vol. 52, no. 11, pp. 65-75, Nov 2014.

[47] Z. Chang, Z. Zhou, S. Zhou, T. Chen, and T. Ristaniemi, "Towards service-oriented $5 \mathrm{~g}$ : Virtualizing the networks for everything-as-aservice," IEEE Access, vol. 6, pp. 1480-1489, 2018.

[48] R. Wireless, "Vodafone, ericsson connect german labs in $5 \mathrm{~g}$ mobile edge poc," http://www.rcrwireless.com/20160701/europe/ germany-5g-mobile-edge-poc-tag17 Tech. Rep., jul 2016, accessed: 2019-07-28

[49] V. K. Singh, H. Chawla, and V. A. Bohara, "A proof-of-concept device-to-device communication testbed," CoRR, vol. abs/1601.01398, 2016. [Online]. Available: http://arxiv.org/abs/1601.01398

[50] M. Shirvanimoghaddam, K. Shirvanimoghaddam, M. M. Abolhasani, M. Farhangi, V. Z. Barsari, H. Liu, M. Dohler, and M. Naebe, "Towards a Green and Self-Powered Internet of Things Using Piezoelectric Energy Harvesting," arXiv e-prints, p. arXiv:1712.02277, Dec 2017.

[51] 3GPP, "Standards for the iot," https://www.3gpp.org/news-events/ 3gpp-news/1805-iot_r14 Tech. Rep., dec 2016, accessed: 2019-0728.

[52] J. F. Monserrat, G. Mange, V. Braun, H. Tullberg, G. Zimmermann4, and O. Bulakci, "Metis research advances towards the $5 \mathrm{~g}$ mobile and wireless system definition," EURASIP Journal on Wireless Communications and Networking, 2015.

[53] P. Arabas, "Energy aware data centers and networks: a survey," Journal of Telecommunications and Information Technology, no. 4, pp. 26-36, 2018.

[54] "Final report on green radio technologies, earth project delivrable d4.3," https://www.ict-earth.eu/publications/deliverables/ deliverables.html accessed: 2017-12-16.

[55] S. Zhang, X. Xu, Y. Wu, L. Lu, and Y. Chen, "A survey on 5g new waveform: From energy efficiency aspects," pp. 1939-1943, Nov 2014.

[56] L. C. Wang and S. Rangapillai, "A survey on green $5 \mathrm{~g}$ cellular networks," in 2012 International Conference on Signal Processing and Communications (SPCOM), July 2012, pp. 1-5.

[57] "Ntt docomo launches eco-friendly cell phone station," http://www. japanfs.org/en/news/archives/news_id025703.html accessed: 201706-28.

[58] R. Garafola, "Eco-sustainable wireless networks: Ready for prime time," Alcatel-Lucent, 2009.

[59] F. Mukhlif, K. A. B. Nooridin, Y. A. AL-Gumaei, and A. S. ALRassas, "Energy harvesting for efficient 5g networks," in 2018 International Conference on Smart Computing and Electronic Enterprise (ICSCEE), July 2018, pp. 1-5.
[60] M. Ashraf, J. W. Jang, J.-K. Han, and K.-G. Lee, "Video multicast cooperative communication in $5 \mathrm{~g}$ systems with radio frequency energy harvesting," Computer Communications, vol. 118 , pp. $60-68,2018$. [Online]. Available: http: //www.sciencedirect.com/science/article/pii/S014036641730508X

[61] " $5 \mathrm{~g}$ ippp (2014) $5 \mathrm{~g}$ infrastructure ppp: The next generation of communication networks will be 'made in eu'," http://ec.europa.eu/information_society/newsroom/cf/dae/ itemdetail.cfm?item_id=14424 accessed: 2017-12-16.

[62] J. Paradiso and T. Starner, "Energy scavenging for mobile and wireless electronics," Pervasive Computing, IEEE, vol. 4, no. 1, pp. 18-27, Jan 2005.

[63] X. Lu, P. Wang, D. Niyato, D. I. Kim, and Z. Han, "Wireless networks with rf energy harvesting: A contemporary survey," Communications Surveys Tutorials, IEEE, vol. 17, no. 2, pp. 757-789, Secondquarter 2015.

[64] S. Devasenapathy, R. Venkatesha Prasad, V. Rao, and I. Niemegeers, "Impact of antenna directionality and energy harvesting rate on neighbor discovery in eh-iots," in Consumer Communications and Networking Conference (CCNC), 2013 IEEE, Jan 2013, pp. 302-307. [Online]. Available: http: //www.es.ewi.tudelft.nl/papers/2013-Devasenapathy-EHND.pdf

[65] A. Radwan and J. Rodriguez, Energy Efficient Smart Phones for $5 G$ Networks, ser. Signals and Communication Technology. Springer International Publishing, 2016. [Online]. Available: https://books.google.co.ma/books?id=jCkTvgAACAAJ

[66] Z. Kausar, A. Reza, M. U. Saleh, H. Saleh, and H. Ramiah, "Energizing wireless sensor networks by energy harvesting systems: Scopes, challenges and approaches," Renewable and Sustainable Energy Reviews, vol. 38, pp. 973-989, 072014.

[67] N. Chughtai, "Energy harvesting in 5g networks," Ph.D. dissertation, 082018.

[68] A. Kurs, K. A.S., R. Moffatt, J. Joannopoulos, P. Fisher, and M. Soljai, "Wireless power transfer via strongly coupled magnetic resonances," Science, Jul 2007.

[69] R. Zhang and C. K. Ho, "Mimo broadcasting for simultaneous wireless information and power transfer," IEEE Transactions on Wireless Communications, vol. 12, no. 5, pp. 1989-2001, May 2013.

[70] C. Mikeka and H. Arai, "Sustainable energy harvesting technologies - past, present and future, hap. design issues in radio frequency energy harvesting system," InTech, 2011.

[71] H. Ju and R. Zhang, "Throughput maximization in wireless powered communication networks," in 2013 IEEE Global Communications Conference (GLOBECOM), Dec 2013, pp. 4086-4091.

[72] T. Kaźmierski and S. Beeby, Energy Harvesting Systems: Principles, Modeling and Applications, ser. SpringerLink : Bücher. Springer New York, 2010. [Online]. Available: https://books.google.co.ma/books?id=e4QQc6AEG1IC

[73] P. EFTHYMAKIS, “A rectenna for 5g energy harvesting," 2014.

[74] M. Maso, Energy Harvesting Oriented Transceiver Design for $5 G$ Networks. Cham: Springer International Publishing, 2016, pp. 67-95.

[75] X. Zhou, R. Zhang, and C. K. Ho, "Wireless information and power transfer: Architecture design and rate-energy tradeoff," IEEE Transactions on Communications, vol. 61, no. 11, pp. 4754-4767, November 2013.

[76] S. Kim, R. Vyas, J. Bito, K. Niotaki, A. Collado, A. Georgiadis, , and M. M. Tentzeris, "Ambient rf energy-harvesting technologies for selfsustainable standalone wireless sensor platforms," in Proc. IEEE, November 2014, pp. 1649-1666.

[77] H. Kanaya, S. Tsukamaoto, T. Hirabaru, D. Kanemoto, R. K. Pokharel, and K. Yoshida, "Energy harvesting circuit on a onesided directional flexible antenna," IEEE Microwave and Wireless Components Letters, vol. 23, no. 3, pp. 164-166, March 2013.

[78] B. Pham and A. Pham, "Triple bands antenna and high efficiency rectifier design for rf energy harvesting at 900, 1900 and $2400 \mathrm{mhz}$,' 06 2013, pp. 1-3. 
[79] L. M. Borges, N. Barroca, H. M. Saraiva, J. Tavares, P. T. Gouveia, F. J. Velez, C. Loss, R. Salvado, P. Pinho, R. Gonçalves, N. B. Carvalho, R. Chavéz-Santiago, and I. Balasingham, "Design and evaluation of multi-band rf energy harvesting circuits and antennas for wsns," in 201421 st International Conference on Telecommunications (ICT), May 2014, pp. 308-312.

[80] N. A. Zainuddin, Z. Zakaria, M. N. Husain, B. M. Derus, M. Z. A. A. Aziz, M. A. Mutalib, and M. A. Othman, "Design of wideband antenna for rf energy harvesting system," in 2013 3rd International Conference on Instrumentation, Communications, Information Technology and Biomedical Engineering (ICICI-BME), Nov 2013, pp. 162-166.

[81] J. SCHNEIDER1, M. MRNKA, J. GAMEC, M. GAMCOVA, and Z. RAIDA, "Vivaldi antenna for rf energy harvesting," RADIOENGINEERING, vol. 25, pp. 666-671, 122016.

[82] "Energy harvesting system market by technology (light, vibration, electromagnetic, thermal), application (building and home automation, consumer electronics, industrial, transportation, security), and geography - global forecast to 2022," http://www.marketsandmarkets. com/Market-Reports/energy-harvesting-market-734.html Apr 2016, accessed: 2019-05-19.

[83] K. hao Liu and P. Lin, "Toward self-sustainable cooperative relays: state of the art and the future," Communications Magazine, IEEE, vol. 53, no. 6, pp. 56-62, June 2015.

[84] J. Morra, "Startup's energy harvesting chips run on radio frequencies," https://www.electronicdesign.com/analog/ startups-energy-harvesting-chips-run-radio-frequencies January 2019, accessed: 2019-05-19.

[85] R. Pell, "Boom time for energy harvesting chip market," https://www.eenewseurope.com/news/ boom-time-energy-harvesting-chip-market-0 September 2018, accessed: 2019-05-19.

[86] A. M. Zungeru, L. Ang, S. R. S. Prabaharan, and K. P. Seng, "Radio frequency energy harvesting and management for wireless sensor networks," CoRR, vol. abs/1208.4439, 2012. [Online]. Available: http://arxiv.org/abs/1208.4439

[87] A. Rajaram, R. Khan, S. Tharranetharan, D. N. Jayakody, R. Dinis, and S. Panic, "Novel swipt schemes for 5g wireless networks," Sensors, vol. 19, p. 1169, 032019.

[88] X. Ge, H. Jia, Y. Zhong, Y. Xiao, Y. Li, and B. Vucetic, "Energy efficient optimization of wireless-powered $5 \mathrm{~g}$ full duplex cellular networks: A mean field game approach," IEEE Transactions on Green Communications and Networking, pp. 1-1, 2019.

[89] S. Mahama, D. K. P. Asiedu, and K. Lee, "Simultaneous wireless information and power transfer for cooperative relay networks with battery," IEEE Access, vol. 5, pp. 13 171-13 178, 2017.

[90] Q. Li, J. Gao, H. Liang, L. Zhao, and X. Tang, "Optimal power allocation for wireless sensor powered by dedicated rf energy source," IEEE Transactions on Vehicular Technology, vol. 68, no. 3, pp. 2791-2801, March 2019.

[91] T. A. Khan, R. W. Heath, and P. Popovski, "Wirelessly powered communication networks with short packets," IEEE Transactions on Communications, vol. 65, no. 12, pp. 5529-5543, Dec 2017.

[92] T. Do, "Optimal energy harvesting scheme for power beacon-assisted wireless-powered networks," Indonesian Journal of Electrical Engineering and Computer Science, vol. 7, pp. 802-808, 092017.

[93] O. Galinina, H. Tabassum, K. Mikhaylov, S. Andreev, E. Hossain, and Y. Koucheryavy, "On feasibility of 5g-grade dedicated rf charging technology for wireless-powered wearables," IEEE Wireless Communications, vol. 23, pp. 28-37, 2015.

[94] A. Nechibvute, A. CHAWANDA, N. Taruvinga, and P. LUHANGA, "Radio frequency energy harvesting sources," Acta Electrotechnica et Informatica, vol. 17, pp. 19-27, 122017.

[95] M. L. Ku, W. Li, Y. Chen, and K. J. R. Liu, "Advances in energy harvesting communications: Past, present, and future challenges," IEEE Communications Surveys Tutorials, vol. 18, no. 2, pp. 1384-1412, Secondquarter 2016.

[96] N. Zhao, F. Yu, and V. Leung, "Wireless energy harvesting in interference alignment networks," Communications Magazine, IEEE, vol. 53, no. 6, pp. 72-78, June 2015.
[97] M. Maso, S. Lakshminarayana, T. Q. S. Quek, and H. V. Poor, "The price of self-sustainability for block transmission systems," IEEE Journal on Selected Areas in Communications, vol. 33, no. 8, pp. 1549-1562, Aug 2015.

[98] W. Shin, M. Vaezi, J. Lee, and H. V. Poor, "Cooperative wireless powered communication networks with interference harvesting," IEEE Transactions on Vehicular Technology, vol. 67, no. 4, pp. 3701-3705, April 2018.

[99] H. Kong, I. Flint, P. Wang, D. Niyato, and N. Privault, "Exact performance analysis of ambient rf energy harvesting wireless sensor networks with ginibre point process," IEEE Journal on Selected Areas in Communications, vol. 34, no. 12, pp. 3769-3784, Dec 2016.

[100] M. L. Memon, N. Saxena, A. Roy, and D. R. Shin, "Backscatter communications: Inception of the battery-free era- a comprehensive survey," Electronics, vol. 8, no. 2, 2019. [Online]. Available: https://www.mdpi.com/2079-9292/8/2/129

[101] H. Choi and W. Shin, "Harvest-until-access protocol based on slotted aloha for wireless powered dense networks," in 2019 International Conference on Electronics, Information, and Communication (ICEIC), Jan 2019, pp. 1-6.

[102] Y. Liu, Y. Zhang, R. Yu, and S. Xie, "Integrated energy and spectrum harvesting for $5 \mathrm{~g}$ wireless communications," Network, IEEE, vol. 29, pp. 75-81, 062015 .

[103] J. Ghosh and Z. Jakó, "Mac protocol operation in energy harvesting for $5 \mathrm{~g}$ networks," ICST Transactions on Mobile Communications and Applications, vol. 3, p. 153554, 012018.

[104] A. Ghazanfari, H. Tabassum, and E. Hossain, "Ambient RF energy harvesting in ultra-dense small cell networks: Performance and trade-offs," CoRR, vol. abs/1512.03122, 2015. [Online]. Available: http://arxiv.org/abs/1512.03122

[105] M. S. C. A. H2020-MSCA-ITN-ETN, "Sustainable cellular network harvesting ambient energy, deliverable $\mathrm{d} 3.1: \mathrm{Wp} 3$ - sustainable networking, d3.1 - intermediate report," http://www.scavenge.eu/ wp-content/uploads/2018/02/D3.1.pdf Tech. Rep., jan 2018, accessed: $2019-07-28$

[106] Z. Chen, Z. Chen, L. X. Cai, and Y. Cheng, "Energy-throughput tradeoff in sustainable cloud-ran with energy harvesting," in 2017 IEEE International Conference on Communications (ICC), May 2017, pp. 1-6.

[107] G. Qiao, S. Leng, Y. Zhang, M. Zeng, and L. Xu, "Multiple timescale energy scheduling with energy harvesting aided heterogeneous cloud radio access networks," in 2016 IEEE/CIC International Conference on Communications in China (ICCC), July 2016, pp. 1-6.

[108] Q. Pham, F. Fang, H. Vu, M. Le, Z. Ding, L. B. Le, and W. Hwang, "A survey of multi-access edge computing in $5 \mathrm{~g}$ and beyond: Fundamentals, technology integration, and state-ofthe-art," CoRR, vol. abs/1906.08452, 2019. [Online]. Available: http://arxiv.org/abs/1906.08452

[109] T. Dlamini, Ángel Fernández Gambín, D. Munaretto, and M. Rossi, "Online supervisory control and resource management for energy harvesting bs sites empowered with computation capabilities," Wireless Communications and Mobile Computing, p. 17, 2019.

[110] Y. Mao, J. Zhang, and K. B. Letaief, "Dynamic computation of floading for mobile-edge computing with energy harvesting devices," IEEE Journal on Selected Areas in Communications, vol. 34, no. 12, pp. 3590-3605, Dec 2016.

[111] H.-S. Nguyen, T.-S. Nguyen, and M. Voznak, "Wireless powered d2d communications underlying cellular networks: design and performance of the extended coverage," Automatika, vol. 58, no. 4, pp. 391-399, 2017. [Online]. Available: https://doi.org/10.1080/00051144.2018.1455016

[112] Y. Liu, L. Wang, S. A. R. Zaidi, M. Elkashlan, and T. Duong, "Secure $\mathrm{d} 2 \mathrm{~d}$ communication in large-scale cognitive cellular networks: A wireless power transfer model," IEEE Transactions on Communications, vol. 64, 112015.

[113] L. S. Mohjazi, M. Dianati, G. K. Karagiannidis, S. Muhaidat, and M. Al-Qutayri, "Rf-powered cognitive radio networks: technical challenges and limitations," IEEE Communications Magazine, vol. 53, no. 4, pp. 94-100, 2015. 
[114] D. Do, M. Vaezi, and T. Nguyen, "Wireless powered cooperative relaying using NOMA with imperfect CSI," CoRR, vol. abs/1810.00276, 2018. [Online]. Available: http://arxiv.org/abs/1810.00276

[115] M. Basharat, "Joint user grouping and time allocation for noma with wireless power transfer," 2017.

[116] P. T. Hiep and T. M. Hoang, "Non-orthogonal multiple access and beamforming for relay network with rf energy harvesting," ICT Express, 2019. [Online]. Available: http: //www.sciencedirect.com/science/article/pii/S240595951930150X

[117] A. Rauniyar, P. E. Engelstad, and O. N. Østerbø, "Rf energy harvesting and information transmission based on noma for wireless powered iot relay systems," in Sensors, 2018.

[118] Y. Zhu, L. Wang, K. Wong, S. Jin, and Z. Zheng, "Wireless power transfer in massive mimo-aided hetnets with user association," IEEE Transactions on Communications, vol. 64, no. 10, pp. 4181-4195, Oct 2016.

[119] R. Hamdi, E. Driouch, and W. Ajib, "Energy management in largescale mimo systems with per-antenna energy harvesting," in 2017 IEEE International Conference on Communications (ICC), May 2017, pp. 1-6.

[120] R. Shrestha and G. Amarasuriya, "Wireless energy harvesting in massive mimo with low-dimensional digital precoding," in Proc.
IEEE Global Communication Conference (GLOBECOM), 12 2018, pp. $1-7$.

[121] T. A. Khan, A. Alkhateeb, and R. W. H. Jr., "Millimeter wave energy harvesting," CoRR, vol. abs/1509.01653, 2015. [Online]. Available: http://arxiv.org/abs/1509.01653

[122] L. Dai, B. Wang, M. Peng, and S. Chen, "Hybrid precoding-based millimeter-wave massive mimo-noma with simultaneous wireless information and power transfer," IEEE Journal on Selected Areas in Communications, vol. 37, no. 1, pp. 131-141, Jan 2019.

[123] M. Wagih, A. S. Weddell, and S. Beeby, "Millimeter-wave textile antenna for on-body rf energy harvesting in future $5 \mathrm{~g}$ networks," in Wireless Power Week 2019, IEEE Wireless Power Transfer Conference. (21/06/19), June 2019, pp. 1-4. [Online]. Available: https://eprints.soton.ac.uk/431955/

[124] N. I. Miridakis and T. A. Tsiftsis, "A new interweave cognitive radio scheme for out-band energy harvesting systems," IEEE Access, vol. 6, pp. 72 225-72 232, 2018.

[125] W. Zhao, R. She, and H. Bao, "Energy efficiency maximization for two-way relay assisted cr-noma system based on swipt," IEEE Access, vol. 7, pp. 72 062-72 071, 2019.

[126] B. Alzahrani and W. Ejaz, "Resource management for cognitive iot systems with rf energy harvesting in smart cities," IEEE Access, vol. 6, pp. 62 717-62 727, 2018. 